\title{
Direct numerical simulation of turbulent scalar transport across a flat surface
}

\author{
H. HERLIN A ${ }^{1} \dagger$, AND J. G. WISSINK \\ ${ }^{1}$ Institute for Hydromechanics, Karlsruhe Institute of Technology, Kaiserstr.12, 76131 \\ Karlsruhe, Germany \\ ${ }^{2}$ School of Engineering and Design, Brunel University London, Kingston Lane, Uxbridge, UB8 \\ $3 \mathrm{PH}, \mathrm{UK}$
}

(Received ?; revised ?; accepted ?. - To be entered by editorial office)

To elucidate the physical mechanisms that play a role in the interfacial transfer of atmospheric gases into water, a series of direct numerical simulations of mass transfer across the air-water interface driven by isotropic turbulence diffusing from below has been carried out for various turbulent Reynolds numbers $\left(R_{T}=84,195,507\right)$. To allow a direct (unbiased) comparison of the instantaneous effects of scalar diffusivity, in each of the DNS up to six scalar advection-diffusion equations with different Schmidt numbers were solved simultaneously. As far as the authors are aware this is the first simulation that is capable to accurately resolve the realistic Schmidt number, $S c=500$, that is typical for the transport of atmospheric gases such as oxygen in water. For the range of turbulent Reynolds numbers and Schmidt numbers considered, the normalized transfer velocity $K_{L}$ was found to scale with $R_{T}^{-1 / 2}$ and $S c^{-1 / 2}$, which indicates that the largest eddies present in the isotropic turbulent flow introduced at the bottom of the computational domain tend to determine the mass transfer. The $K_{L}$ results were also found to be in good agreement with the surface divergence model of McCready, Vassiliadou \& Hanratty (AIChE J., vol. 32, 1986, pp. 1108-1115) when using a constant of proportionality of 0.525. Although close to the surface large eddies are responsible for the bulk of the gas transfer, it was also observed that for higher $R_{T}$ the influence of smaller eddies becomes more important.

Key words: turbulent mixing, air/sea interactions, computational methods

\section{Introduction}

Many environmentally important gases like oxygen $\left(\mathrm{O}_{2}\right)$, carbon dioxide $\left(\mathrm{CO}_{2}\right)$, nitrous oxide (NO) etc. have a very low diffusivity in water. Because of this, the mass transfer of these gases across the water surface (high Schmidt number process) is governed by resistance on the liquid side and the typical thickness $\delta$ of the gas-saturated layer immediately below the water surface is very small $(\delta \approx 10-1000 \mu \mathrm{m})$. A wide variety of interfacial gas exchange processes can be found in nature, including oxygen absorption from the atmosphere into streams or lakes (reaeration), $\mathrm{CO}_{2}$ absorption by the ocean (carbon sinks) or the volatilization of pollutants. Even though the equations that describe fluid flow and scalar transport (Equation 2.1) are well-known, the very small thickness

$\dagger$ Email address for correspondence: herlina.herlina@kit.edu 
of the concentration boundary layer, which is thinner than the Kolmogorov scale of the liquid flow, complicates the effort of understanding the actual gas transfer mechanisms.

Early studies focused on developing conceptual models and finding empirical relations between measurable flow quantities and the global gas transfer velocity

$$
K_{L}=\frac{j}{c_{s}-c_{b}}
$$

where $j$ is the average gas flux, $c_{b}$ and $c_{s}$ are the average gas concentrations in the bulk and at the surface of the water, respectively. The oldest model is the film-model of Lewis \& Whitman (1924) who assumed the presence of a stagnant film on both sides of the interface in which the gas transfer is controlled by molecular diffusion, $D$. This assumption led to the equation $K_{L}=D / \delta$, in which $\delta$ is the thickness of the stagnant film. In various experiments (see e.g. McCready et al. 1986) this linear relationship between $K_{L}$ and $D$ was found to be an oversimplification of the actual transfer process. Higbie (1935) realised that the actual transfer velocity was strongly dependent on the rate at which turbulence replaced the saturated fluid near the interface by fresh fluid from below. To describe this he introduced a (constant) surface-renewal time, $T$, in his penetration model. Danckwerts (1951) subsequently improved Higbies model by allowing the surface renewal rate, $r$, to follow an exponential distribution so that $T$ was no longer a constant. As a result he obtained the relationship $K_{L} \approx \sqrt{D r}$. The hydrodynamics are described by the renewal rate $r$, which needs to be determined experimentally. Some researchers tried to circumvent the experimental determination of $r$ by relating it to measurable flow parameters. By assuming that the surface renewal rate is determined by the largest turbulent eddies in a flow, Fortescue \& Pearson (1967) estimated $r$ by $u_{r m s} / L_{\infty}$, where $u_{r m s}$ is the rms of the turbulent fluctuations and $L_{\infty}$ is the integral length scale of the turbulence. Hence, in this so-called large-eddy model $K_{L}$ is given by $K_{L} \approx \sqrt{D u_{r m s} / L_{\infty}}$, or alternatively $K_{L} S c^{1 / 2} / u_{r m s} \propto R_{T}^{-1 / 2}$. An alternative approach for the determination of $r$ is given by the small-eddy model developed by Banerjee et al. (1968) and Lamont $\&$ Scott (1970). By assuming that the small eddies are determining the surface renewal rate, they approximated $r$ by $\sqrt{\varepsilon / \nu}$, where $\varepsilon$ is the turbulent dissipation rate near the surface and $\nu$ is the kinematic viscosity. As a result they obtained the equation $K_{L} \approx$ $\sqrt{D}[\varepsilon / \nu]^{1 / 4}$ or $K_{L} S c^{1 / 2} / u_{r m s} \propto R_{T}^{-1 / 4}$. Theofanous et al. (1976) subsequently proposed two regimes, in which the large-eddy model is valid for low $R_{T}$ and the small-eddy model is valid for high $R_{T}$, where the critical $R_{T}$ is approximately 500 . Finally, by performing numerical investigations, McCready et al. (1986) found that the surface divergence plays an important role in the interfacial gas transfer process. The main problem of conceptual models (reviews of models can be found in e.g. Theofanous (1984); Jähne \& Haussecker (1998)) is to find a reliable relation between the near surface hydrodynamics and the global (in the bulk) turbulent flow parameters. The drawback of empirical relations (see for instance O'Connor \& Dobbins 1956; Plate \& Friedrich 1984; Moog \& Jirka 2002) often is the limited applicability of locally developed equations to other flow conditions.

To date, improved knowledge of the physical mechanisms of the gas transfer process has been obtained through detailed mapping of both the gas concentration and the fluid flow near the surface, which was made possible by the fast development of laboratory measurement techniques as well as increased numerical computing capabilities. Non-intrusive optical measurement techniques such as Particle Image Velocimetry (PIV) have been used in order to understand the near surface hydrodynamics (e.g. Tamburrino \& Gulliver 2002; Banerjee et al. 2004; McKenna \& McGillis 2002, 2004b; Sugihara \& Tsumori 2005; Turney \& Banerjee 2013), while Laser Induced Fluorescence (LIF) enabled visualization of the gas concentration fields (e.g Wolff \& Hanratty 1994; Münsterer 
et al. 1995; Schladow et al. 2002; Woodrow \& Duke 2002; Herlina \& Jirka 2004; Walker \& Peirson 2008). Various research groups (such as Lu \& Hetsroni (1995); Handler et al. (1999); Nagaosa (1999); Yamamoto et al. (2001)) conducted direct numerical simulations (DNS) of passive heat/mass transfer across the free surface of an open-channel flow. These studies show a number of important features such as the correlation between the vortices ejected from the bottom region and the near-surface concentration field. Magnaudet \& Calmet (2006) used a large-eddy simulation (LES) to perform a statistical analysis of the structure of the near-surface region of an open-channel flow at a high Reynolds number. Kermani \& Shen (2009) used DNS to study characteristics of interfacial mass transfer driven by free-surface turbulence. They investigated the quantification of the surface age related to the surface renewal models pioneered by Higbie's penetration theory (Higbie 1935) and further elaborated by Danckwert's random surface renewal model (Danckwerts 1951). These models assume that the turbulence in the bulk region transports fluid with a low gas-saturation up to the surface where gas transfer takes place. The surface age can be thought of as the time between two surface renewal events. Kermani \& Shen (2009) concluded that Higbie's model is inappropriate for describing the gas transfer and Danckwert's model is only applicable at large surface age where the gas transfer is actually insignificant. Hasegawa \& Kasagi (2009) performed a hybrid DNS/LES calculation of a coupled air-water turbulent flow and associated mass transfer for $S c=1$ and 100 at $R e_{\tau}=150$ ( $R e_{\tau}$ is the Reynolds number based on the interfacial friction velocity). They focused on the relation between the local interfacial mass transfer and the surface divergence and proposed a criterion for the validity of the surface divergence model. Recently, Khakpour et al. (2011) performed low $S c$ DNS with turbulent shear flow and examined the effect of surfactant contamination at the surface on the mass transfer process. The scalar statistics near the contaminated surface were found to behave similarly to those near a solid wall. In this paper, we focus on examining the effect of $\operatorname{Schmidt}(S c)$ and turbulent Reynolds $\left(R_{T}\right)$ numbers on mass transfer at a clean flat-interface using DNS performed at a wide range of $S c$ (1 to 500$)$ and low to moderate $R_{T}$ (84 to 507 ).

The numerical setup is chosen largely in accordance with the experimental work of Herlina \& Jirka (2008). In these experiments the gas transfer process across the airwater interface driven by grid-stirred turbulence (a convenient analogy to bottom-shear induced turbulence) in the water phase was investigated using PIV and LIF techniques (see Figure 1). The synoptic visualization of velocity and concentration fields near the interface provided a better insight into the transfer mechanisms. Direct quantification of the molecular diffusive transport $D \partial \bar{c} / \partial z$ and the turbulent mass flux $\overline{c^{\prime} w^{\prime}}$ in terms of the total mass flux $\bar{j}$

$$
\bar{j}=\overline{c^{\prime} w^{\prime}}-D \partial \bar{c} / \partial z
$$

in the vertical direction were made possible. Their data provided experimental evidence that the normalized mean $\overline{c^{\prime} w^{\prime}}$ profile increases from around 0 at the water surface to 1 within a short distance below the surface (approx. twice the boundary layer thickness). The techniques applied, however, still face difficulties in resolving the uppermost diffusive sublayer as well as any structures present in the deeper bulk region.

With the present DNS we aim to complement the laboratory results and obtain details of the gas transfer mechanisms that in the laboratory experiments might be affected by limitation of measurement accuracy and/or unavoidable disturbances (such as unwanted surface contamination). As in the experiments, we focused on elucidating the transfer process in the near surface region at various turbulent Reynolds numbers $R_{T}$ ranging from low to moderate. Another focus of the present numerical study is to complement the laboratory results by studying the effect of varying the Schmidt numbers $S c$, defined 
FiguRE 1. Schematic illustration depicting the gas transfer problem driven by isotropic turbulence diffusing from below (a convenient analogy to bottom-shear induced turbulent flow.)

as the ratio of the momentum diffusivity (viscosity $\nu$ ) and mass diffusivity $D$. Typical Schmidt numbers for gases dissolved in water are $S c \approx 200$ for Helium, $S c \approx 500$ for oxygen and $S c \approx 600$ for carbon dioxide. Due to limitations in computing capacity, most previous DNS studies were limited to Schmidt numbers smaller than 10 and considered typically only one turbulent Reynolds number. To be able to perform the highly accurate simulations at realistic Schmidt numbers, a numerical code especially developed for this purpose was used. It uses a special discretization of the scalar advection using a weighted, essentially non-oscillatory (WENO) scheme (Liu et al. 1994) that is able to resolve steep concentration gradients efficiently together with a fourth-order discretisation of scalar diffusion and a fourth-order incompressible flow solver. To further increase the accuracy of the scalar discretisation a dual meshing strategy was used with a finer mesh for the scalar than for the flow field. The code was parallelised so that it runs on modern, massively parallel supercomputers. As far as the authors are aware these are the first direct numerical simulations of mass transfer across the air-water interface in which the scalar transport at Schmidt numbers up to $S c=500$ is fully resolved for low to moderate $R_{T}$.

\section{Description of Computations}

The problem under consideration is the gas transfer across the air-water interface driven from below by a homogeneous turbulent flow that is generated at the bottom of the water body. The passive scalar transport is governed by the three-dimensional convection diffusion equation of the scalar $c=c(x, y, z, t)$ that in conservative form reads

$$
\frac{\partial c}{\partial t}+\frac{\partial u c}{\partial x}+\frac{\partial v c}{\partial y}+\frac{\partial w c}{\partial z}=\frac{1}{R e S c}\left(\frac{\partial^{2} c}{\partial x^{2}}+\frac{\partial^{2} c}{\partial y^{2}}+\frac{\partial^{2} c}{\partial z^{2}}\right)
$$

where $x, y$ are the horizontal directions and $z$ is the vertical direction, $u, v$ and $w$ are the velocities in the $x, y$, and $z$ directions, $R e$ is the Reynolds number and $t$ denotes time. The fluid motion is governed by the incompressible Navier-Stokes equations where the 
continuity equation reads,

$$
\frac{\partial u}{\partial x}+\frac{\partial v}{\partial y}+\frac{\partial w}{\partial z}=0
$$

and the momentum equations are given by

$$
\begin{gathered}
\frac{\partial u}{\partial t}+\frac{\partial u u}{\partial x}+\frac{\partial u v}{\partial y}+\frac{\partial u w}{\partial z}=-\frac{\partial p}{\partial x}+\frac{1}{R e}\left\{\frac{\partial^{2} u}{\partial x^{2}}+\frac{\partial^{2} u}{\partial y^{2}}+\frac{\partial^{2} u}{\partial z^{2}}\right\} \\
\frac{\partial v}{\partial t}+\frac{\partial v u}{\partial x}+\frac{\partial v v}{\partial y}+\frac{\partial v w}{\partial z}=-\frac{\partial p}{\partial y}+\frac{1}{R e}\left\{\frac{\partial^{2} v}{\partial x^{2}}+\frac{\partial^{2} v}{\partial y^{2}}+\frac{\partial^{2} v}{\partial z^{2}}\right\} \\
\frac{\partial w}{\partial t}+\frac{\partial w u}{\partial x}+\frac{\partial w v}{\partial y}+\frac{\partial w w}{\partial z}=-\frac{\partial p}{\partial z}+\frac{1}{R e}\left\{\frac{\partial^{2} w}{\partial x^{2}}+\frac{\partial^{2} w}{\partial y^{2}}+\frac{\partial^{2} w}{\partial z^{2}}\right\}
\end{gathered}
$$

where $p$ is pressure.

For the simulations, the full set of governing equations mentioned above was solved using a three-dimensional version of our in-house developed code described in Kubrak et al. (2013). The solver was developed specifically with the aim to resolve the details of low-diffusivity scalar transport, which is governed by a very thin boundary layer at the air-water interface. In this code, a fifth-order accurate WENO scheme (Liu et al. 1994) for the scalar convection combined with a fourth-order accurate central method for scalar diffusion were applied in order to accurately capture high concentration gradients. The time-integration for the scalar was performed using a three-stage Runge-Kutta scheme. For the fluid flow, a central finite-difference approach with a fourth-order accurate discretization of the diffusion and a fourth-order-accurate kinetic energy conserving discretization of the convection (Wissink 2004) was used to solve the incompressible 3D Navier-Stokes equations. The spatial discretization of the fluid flow was performed on a non-uniform mesh using a staggered variable arrangement and was combined with a second order accurate Adams-Bashforth method for the time-integration. The Poisson equation for the pressure, which is obtained after substituting the discretized momentum equation into the continuity equation, was solved using a conjugate gradient solver with a simple diagonal preconditioning.

As the scalar diffusivity is much smaller than the momentum diffusivity, significantly more grid points are needed to accurately resolve the evolution of scalar. To deal with this in a computationally efficient manner a dual-meshing strategy was employed in which the scalar concentration field was solved on a finer mesh than the velocity field.

As mentioned above, the setup of the computational domain was in accordance with the laboratory experiments conducted by Herlina \& Jirka (2008). The experiments were performed in a $50 \times 50 \times 45 \mathrm{~cm}^{3}$ water column and the turbulence was generated by an oscillating mesh in the lower part of the tank. To save computing time, only a small part of the fluid domain adjacent to the surface was modeled and periodic boundary conditions were applied in the horizontal directions ( $x$ and $y$ directions) to account for the significantly larger horizontal size of the domain as illustrated in Figure 2. In the vertical direction $(z)$, a symmetry boundary condition was applied at the top of the simulation volume, while at the bottom a Dirichlet boundary condition was used to introduce the background turbulent flow-field. In the simulations, the gravity and / or capillary wave effects induced by the turbulent motions at the water surface were assumed to be negligible so that a rigid lid, shear-free surface approximation could be employed. Note that in all our simulations, the Froude number was of the order of $10^{-3}$ which is very close to zero and thus validates the application of the rigid lid assumption.

While the turbulence in the experiments was generated by an oscillating mesh in the lower part of the tank, in order to save computing time we chose to introduce nearly 


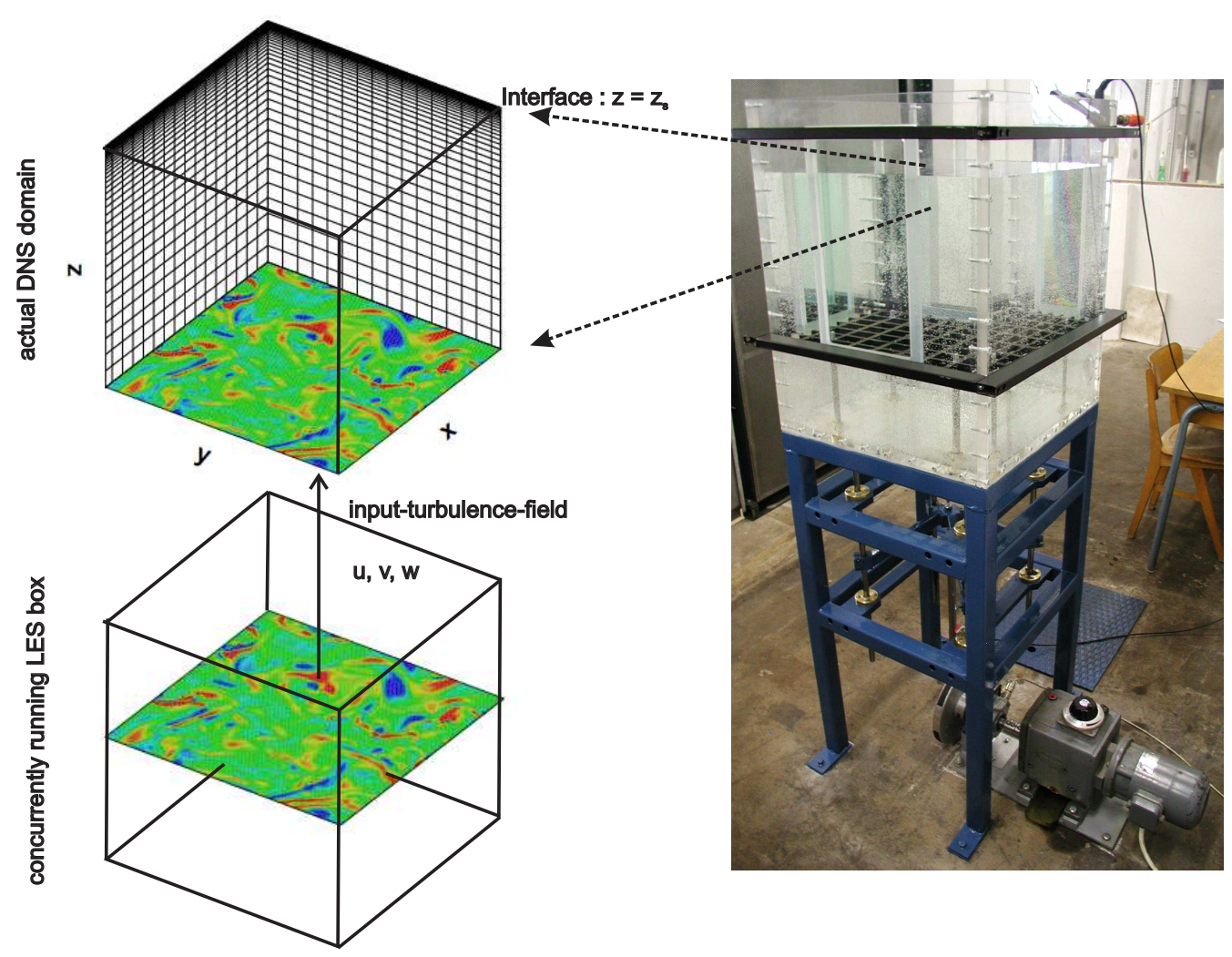

Figure 2. Setup of the computational domain. Only a small part near the surface is modeled. The grid-distribution (only every $8^{\text {th }}$ grid-points) for case GS200 is shown.

isotropic turbulence (grid-like-turbulence) at the bottom of the computational domain. The actual way by which the turbulence at the bottom of the domain is generated is not crucial as long as the near surface hydrodynamics has similar characteristics as those found in the far-field of grid-generated turbulence. In the simulations, the incoming turbulent fluctuations $\left(u^{\prime}, v^{\prime}, w^{\prime}\right)$ stemmed from an LES of isotropic turbulence in a periodic box that ran concurrently with the main DNS. The isotropic turbulence generated in the LES evolved from an initially random flow field that was allowed to develop until a statistically steady state was obtained. The desired level of turbulence was maintained by regularly rescaling the fluctuating velocities. The integral length scale that can be obtained in this way was constrained by the size of the LES domain. The horizontal cross-section of the LES and the DNS domain was identical. At each time-step the instantaneous velocity field in a horizontal plane from the LES was interpolated to the DNS grid (Figure 2) and subsequently used as the bottom boundary condition for the DNS velocity field. After inputing the LES turbulence at the bottom, the small scales that were absent in the LES solution quickly reappeared in the DNS by non-linear transportation of kinetic energy from the larger scales to the smallest scales (thereby completing the spectrum as shown in Figure 7e).

The concentration $c$ was non-dimensionalized by $c^{*}=\left(c-c_{b, 0}\right) /\left(c_{s, 0}-c_{b, 0}\right)$, where $c_{s, 0}$ and $c_{b, 0}$ denote the initial scalar concentration at the surface and in the bulk, respectively. In the remainder of this paper, we will use $c$ to refer to the non-dimensional concentration. The initial value of $c$ was set to zero in the entire computational domain, except close to 


$\begin{array}{lccccc}\text { Run } & S c & \text { Domain } & \text { Mesh Size } & f_{R S} & R_{T} \\ \text { GS80 } & 2-32 & 5 L \times 5 L \times 5 L & 128 \times 128 \times 300 & 1 & 84 \\ \text { GS200 } & 2-32 & 5 L \times 5 L \times 3 L & 128 \times 128 \times 212 & 1 & 195 \\ \text { GS500 } & 2-32 & 20 L \times 20 L \times 5 L & 512 \times 512 \times 300 & 1 & 507 \\ \text { GS80R5 } & 500 & 5 L \times 5 L \times 5 L & 128 \times 128 \times 300 & 5 & 84 \\ \text { GS200R5 } & 500 & 5 L \times 5 L \times 3 L & 128 \times 128 \times 212 & 5 & 195\end{array}$

TABLE 1. Overview of the simulations. Note: The reference length scale $L$ was of the order of $1 \mathrm{~cm}$ in the physical setup. $f_{R S}=$ refinement factor for scalar mesh, $R_{T}$ is the characteristic turbulent Reynolds number of the system defined in Section 4.

the interface where it was defined by

$$
c(\zeta, t)=1-\operatorname{erf}\left(\frac{\zeta}{\sqrt{4 D t_{d}}}\right),
$$

in which $\zeta$ is the distance from the interface and $t_{d}$ is diffusion time. Using this analytical expression as an initial condition and keeping the concentration at the surface saturated $(c=1)$ at all times, the diffusive transport was forced. At the bottom, a Neumann boundary condition $\partial c / \partial z=0$ (zero flux) was employed. This assumption holds as the scalar distribution in the lower part of the computational domain will be nearly fully mixed because of the intense turbulent motions. The distance to the bottom was deemed to be sufficient to ensure that the gas transfer in the region of interest (i.e. in the near surface region) would not be affected by the zero flux boundary condition applied at the bottom.

For grid-stirred or isotropic turbulence driven flow, a convenient measure to match simulated with experimental flow conditions is the turbulent Reynolds number $R_{T}$ (cf. Equation 4.1). Simulations were conducted for three $R_{T}$, using computational domain sizes of $5 L \times 5 L \times 5 L, 5 L \times 5 L \times 3 L$ and $20 L \times 20 L \times 5 L$, respectively. The reference length scale $L$ was typically of the order of $1 \mathrm{~cm}$ and - because the viscosity in all simulations was set to $\nu=1 / 600 U L$ - the reference velocity scale was $U=\frac{\nu_{\text {water }}}{1 / 600} / L=6 \mathrm{~cm} / \mathrm{s}$, where $\nu_{\text {water }}=10^{-2} \mathrm{~cm}^{2} / \mathrm{s}$. The simulations were performed on meshes with $5 \times 10^{6}, 3.5 \times 10^{6}$ and $79 \times 10^{6}$ grid points, respectively, as listed in Table 1 . In all cases, the domain had a uniform grid size in the $x$ and $y$ directions and a slightly stretched grid distribution in the vertical direction to achieve a denser grid near the interface.

The scalar transport (mass transfer) was modeled by solving the advection-diffusion transport equation for several passive scalars concurrently with the time-dependent Navier-Stokes equations. A major benefit is that it allows a detailed comparative study of the effect of the mass diffusivity on the instantaneous distribution of gases in the turbulent flow field. In this study, the mass transfer for six different Schmidt numbers $(2,4$, $8,16,32$ and 500) was simulated. The cases $S c=2$ to 32 were solved simultaneously in a single run on the standard mesh. This allowed us to investigate the influence of the $S c$ number on instantaneous mass transfer driven by exactly the same background turbulent flow.

Separate runs were performed in which the dual-mesh strategy was employed to resolve the low-diffusivity mass transfer at $S c=500$. A refinement factor of 5 was used for the scalar meshes in GS80R5 and GS200R5 (see Table 1). To allow a direct comparison to the other lower $S c$ cases, the simulations GS80R5 and GS200R5 were started using the same initial flow-fields as used in the GS80 and GS200 cases, respectively.

As mentioned above, initially the concentration was set to $c=0$ except close to the 


\begin{tabular}{|c|c|c|c|c|c|c|c|c|c|}
\hline Run & $\begin{array}{l}\Delta z \\
(L)\end{array}$ & $\begin{array}{c}\bar{\Delta} / \pi \\
(L)\end{array}$ & $\stackrel{\eta}{(L)}$ & $S c=2$ & $S c=4$ & $\begin{array}{c}L_{B}(L) \\
S c=8\end{array}$ & $S c=16$ & $S c=32$ & $S c=500$ \\
\hline GS80 & 0.0011 & 0.0038 & 0.1418 & 0.1003 & 0.0709 & 0.0501 & 0.0354 & 0.0251 & - \\
\hline GS200 & 0.0011 & 0.0038 & 0.0489 & 0.0346 & 0.0245 & 0.0173 & 0.0122 & 0.0086 & - \\
\hline GS500 & 0.0011 & 0.0038 & 0.0597 & 0.0422 & 0.0298 & 0.0211 & 0.0149 & 0.0105 & - \\
\hline GS80R5 & 0.00022 & 0.00076 & 0.1418 & - & - & - & - & - & 0.0063 \\
\hline GS200R5 & 0.00022 & 0.00076 & 0.0489 & - & - & - & - & - & 0.0022 \\
\hline
\end{tabular}

TABLE 2. Comparison of the vertical and mean-width grid spacing with the Kolmogorov and Batchelor scales. In all cases, the chosen grid spacing fulfills the Grötzbach criterion. $\eta$ and $L_{B}$ are the estimated thicknesses of the Kolmogorov (see Section 4) and Batchelor (see Section 5.2) sublayers, respectively. The reference length scale $L$ was typically of the order of $1 \mathrm{~cm}$.

interface where $c$ was defined by Equation 2.6 using $t_{d}=30,30,7,7$, and 10 time-units for the cases GS80, GS80R5, GS200, GS200R5, and GS500, respectively.

\section{Refinement Study}

It is well known that an extremely fine grid resolution is needed to capture the dynamics of the turbulent mass transfer at high Schmidt numbers (Grötzbach 1983). To mitigate this, we decided to employ the WENO scheme for scalar convection in order to capture the steep gradients (like capturing shocks in incompressible flow simulations) that might occur anywhere in the computational domain due to the extremely low scalar diffusivity. Capturing such steep gradients does not necessarily mean they need to be fully resolved. Instead, WENO schemes allow the usage of relatively coarse meshes while still capturing the steepest gradients without the over and undershoot that typically occurs in such situations when using spectral methods (Gibbs phenomenon). A detailed validation of the solver's performance and accuracy can be found in Kubrak et al. (2013). In this section we will verify the adequacy of the chosen grid resolution in the present DNS domain (upper box in Figure 2) for both velocity and scalar fields.

To establish which grid resolution would be sufficient we used the Grötzbach criterion (Grötzbach 1983) as a first guideline and subsequently performed grid refinement studies to further verify our choice for the meshes employed in our simulations. As shown in Table 2 the grid spacings used in the upper part of the computational domain in all our simulations fulfill the Grötzbach criterion: The vertical grid resolution near the interface $\Delta z$ is finer than the Batchelor scale (in our case there were at least 7 grid points in the vertical direction within the estimated Batchelor sublayer $L_{B}$ ) and the geometric mean of the grid cells $(\bar{\Delta}=\sqrt[3]{\Delta x \times \Delta y \times \Delta z})$ in the upper part of the computational domain fulfills :

$$
\bar{\Delta} \leqslant \pi L_{B} \quad \text { for } \quad S c \geqslant 1 .
$$

For the velocity, we only carried out a grid refinement test for the case GS80 which has the highest input turbulence intensity and the smallest integral length scale. Because of this, GS80 was deemed to be the most challenging flow problem to resolve and any mesh that is fine enough to accurately resolve the velocity field for this case is expected to be also fine enough for usage in the other cases considered.

The resulting flow-field of a simulation with the base mesh of $128 \times 128 \times 300$ grid points was compared with the one obtained using $176 \times 176 \times 420$ grid points. Snapshots of the 
a)

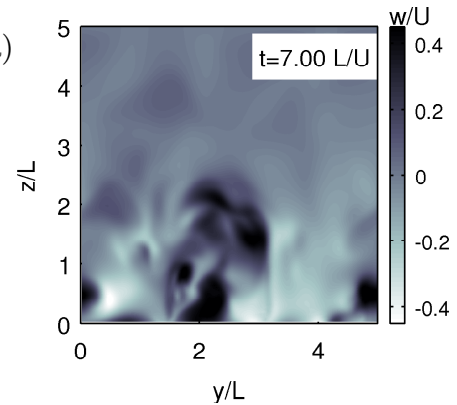

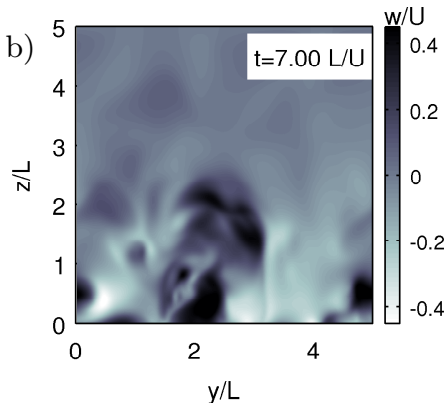

c)

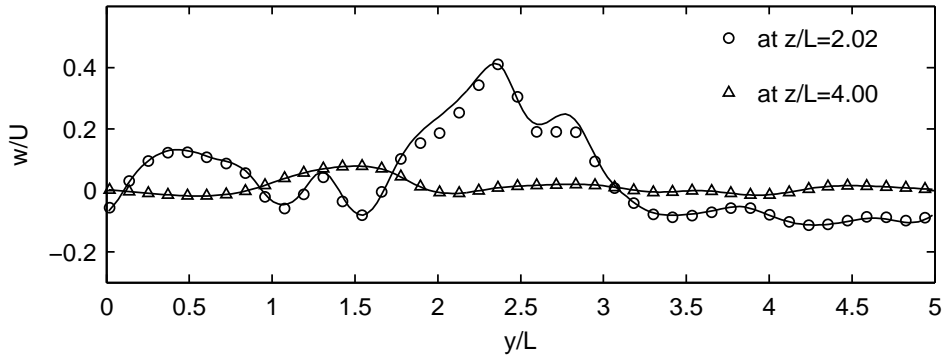

FiguRE 3. Verification of grid resolution of flow field in case GS80 at $x / L=2.5$. Contour plot of the vertical velocity component $w / U$ at $t=7 L / U$ on (a) the standard $128 \times 128 \times 300$ mesh and (b) the refined $176 \times 176 \times 420$ mesh. (c) Instantaneous $w / U$-profiles extracted at $z / L=2.02$ and $z / L=4.00$.

$w$ flow-field for the base and refined meshes are shown in Figures $3 \mathrm{a}$ and $\mathrm{b}$, respectively. After $t=7$ time-units of simulation, both the vertical velocity contours and the velocity profiles at $z / L=2.02$ and $z / L=4.00$ obtained on the standard $128 \times 128 \times 300$ mesh were found to be in good agreement with those obtained on the refined $176 \times 176 \times 420$ mesh. Because the turbulence in the DNS domain is sensitively dependent on the boundary conditions, after some time the velocity fields in the base-line and the refined simulations will diverge. However, as long as the turbulence characteristics of the instantaneous flow field introduced at the bottom for both the base-line and refined DNS domains do not differ significantly, the characteristics of the turbulence inside the DNS boxes should also remain in good agreement. This was indeed found to be true, even after $t=40$ timeunits the spectra of the instantaneous flow-field at various $z$ locations obtained from the standard and refined mesh simulations showed a very similar distribution. Based on this, we concluded that the flow-field on the standard mesh was well resolved.

The grid resolution of the concentration field was tested by employing the dual-mesh option of the code. This means that for the refined case, the flow-field was still solved on the base mesh, while the scalar field was solved on a finer mesh. The scalar-mesh refinement test was performed at $S c=32$ for all three turbulent Reynolds numbers $R_{T}$ (cases GS80, GS200, GS500). Here, the refinement test of the most challenging case only (GS200, see Table 2) is presented.

The results shown in Figure 4 were obtained by starting both the unrefined $(128 \times 128 \times$ 300 grid points $)$ and refined $(256 \times 256 \times 600$ grid points $)$ simulations from the same initial scalar field using the same background turbulent flow-field. Both unrefined and refined 
a)

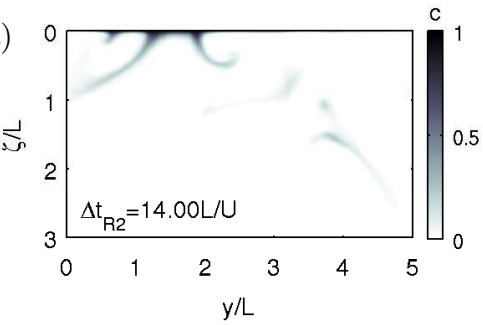

b)

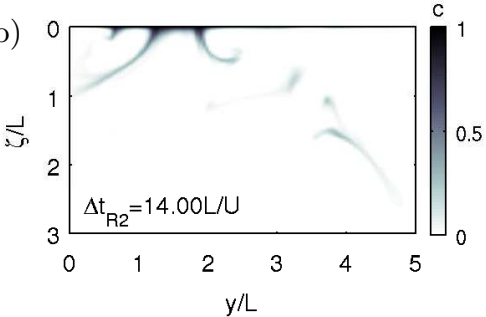

c)

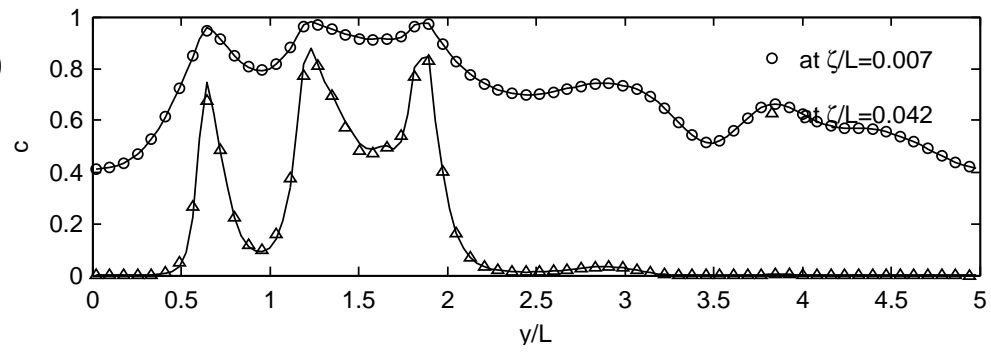

d)
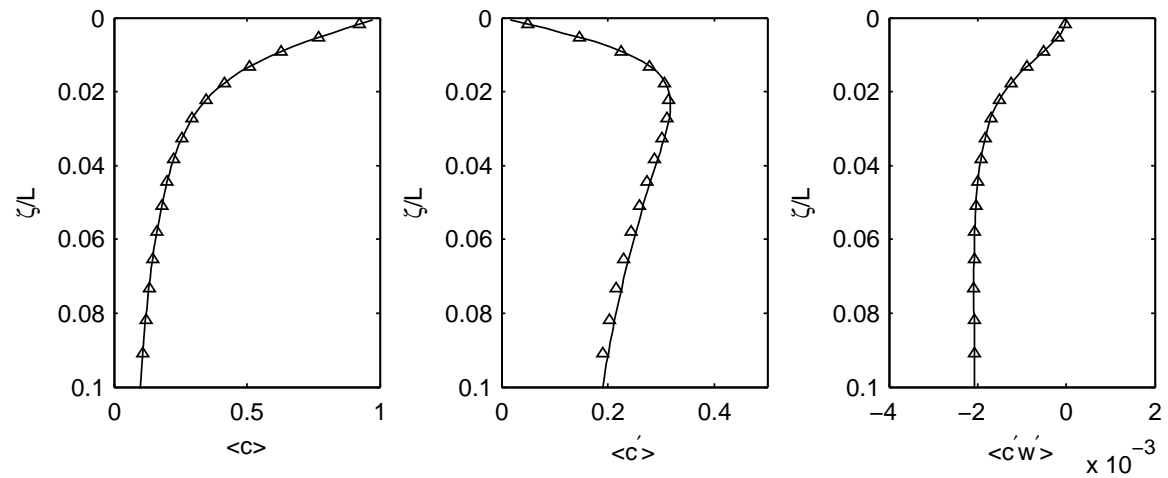

FIGURE 4. Verification of scalar grid spacing for case GS200 with $R_{T}=195$ and $S c=32$. Concentration field at time $\Delta t_{R 2}=14 L / U$ using the (a) standard mesh and (b) refined-mesh with factor 2. (c) Extracted $c$-profiles: $\circ$ using standard mesh at $\zeta / L=0.007, \triangle$ using standard mesh at $\zeta / L=0.042$, - using refined mesh. (d) Spatially averaged $c, c^{\prime}, c^{\prime} w^{\prime}$-profiles at time $\Delta t_{R 2}=14 L / U: \triangle$ standard mesh, - refined mesh. Only every $3^{\text {rd }}$ data point is shown.

simulations were allowed to evolve for the same time period of $\Delta t_{R 2}=14.0$ time-units to check the accuracy of the scalar evolution obtained on the unrefined mesh. The snapshots of the concentration fields in Figures $4 \mathrm{a}$ and $\mathrm{b}$ show a very good agreement between the results obtained using the unrefined and refined meshes. It can be seen that even in the bulk region (outside the region of interest) where the grid becomes relatively coarse, the WENO scheme is still capable of capturing steep gradients in the scalar distribution without introducing any under/overshoots. In the area of interest (immediately below the air-fluid interface), for all simulations a very good agreement was observed between the results obtained on refined and unrefined meshes (see Figure 4c). This is further 


$\begin{array}{lccccc}\text { Run } & R_{T} & L_{\infty} / L & u_{\infty} / U & T_{\infty} L / U & t / T_{\infty} \\ \text { GS80 } & 84 & 1.967 & 0.0359 & 110 & 1.2 \\ \text { GS200 } & 195 & 1.276 & 0.1276 & 20 & 4 \\ \text { GS500 } & 507 & 3.187 & 0.1328 & 48 & 1.5\end{array}$

TABLE 3. Flow parameters. $T_{\infty}$ is the time scale of the large eddies $\left(T_{\infty}=2 L_{\infty} / u_{\infty}\right), t / T_{\infty}$ is the total time over which ensemble averaging is performed in large eddy turnover time scale. The reference length and velocity scales were typically $L=1 \mathrm{~cm}$ and $U=6 \mathrm{~cm} / \mathrm{s}$, respectively.

evidenced by the statistics that were obtained in the region of interest which show a nearly perfect match (Figure 4d). To conclude, in all cases we could verify that the present grid resolution is sufficient to accurately resolve the mass transfer problem in the interface region.

\section{Flow parameters}

The mass transfer simulations reported here were performed at three different turbulent Reynolds numbers $\left(R_{T}=84,195,507\right)$. We used the conventional turbulent Reynolds number definition for grid-stirred turbulence (e.g. Hopfinger \& Toly 1976; Brumley \& Jirka 1987) given by

$$
R_{T}=u_{\infty} 2 L_{\infty} / \nu
$$

where $u_{\infty}$ and $2 L_{\infty}$ are the characteristic velocity and length scales, respectively and $\nu$ is the kinematic viscosity. The characteristic turbulence scalings of the flow-field including the definition of $u_{\infty}$ and length scale $2 L_{\infty}$ used in Equation 4.1 are described below and summarized in Table 3.

To obtain suitable initial flow-fields for the mass transfer simulations, in precursor simulations (without scalar mass transport) the turbulent fluctuations introduced at the bottom of the computational domain were allowed to diffuse upwards and fill the entire computational domain. Only after the flow in the DNS domain was fully developed, the actual mass transfer was activated. Figures 5 to 7 show the statistics of the flow-field used in the mass transfer simulations of the runs with the lowest and highest $R_{T}$ (GS80 and GS500). The plots show time-space averaged values, i.e. space-averaged over the horizontal planes and then time-averaged over $t / T \approx 1$ to 4 large eddy turnover times.

The flow behaviour in the present DNSs was, as aimed, similar to that found in the far-field of grid-generated turbulence. Figure 5 shows the typical decay of the root mean squared turbulent velocities $u_{\mathrm{rms}}, v_{\mathrm{rms}}$ and $w_{\text {rms }}$ with increasing distance away from the turbulence generation source (note that the ordinate $\zeta / L$ denotes the distance from the surface and not from the turbulence source, i.e. $\zeta / L=5$ is the bottom of the DNS domain). The plots also show that the input velocities $u_{r m s}, v_{r m s}, w_{r m s}$ at the bottom of the DNS domain are nearly isotropic and that the homogeneity of the horizontal components $u_{r m s}$ and $v_{r m s}$ is reasonably maintained throughout the whole DNS domain. It should be noted that a much better agreement of the $u_{r m s}$ and $v_{r m s}$ profiles in the bulk region of the DNS domain is obtained when averaging over a much longer period than presented here. For consistency reasons, the period of averaging used for the $u_{r m s}$ and $v_{r m s}$ profiles shown in Figure 5 was chosen to be identical to the period over which the other (scalar) statistics were gathered. In the GS500 case (Figure 5b), for example, we limited the statistical evaluation of the mass transfer simulation to 1.5 large-eddy 
a)

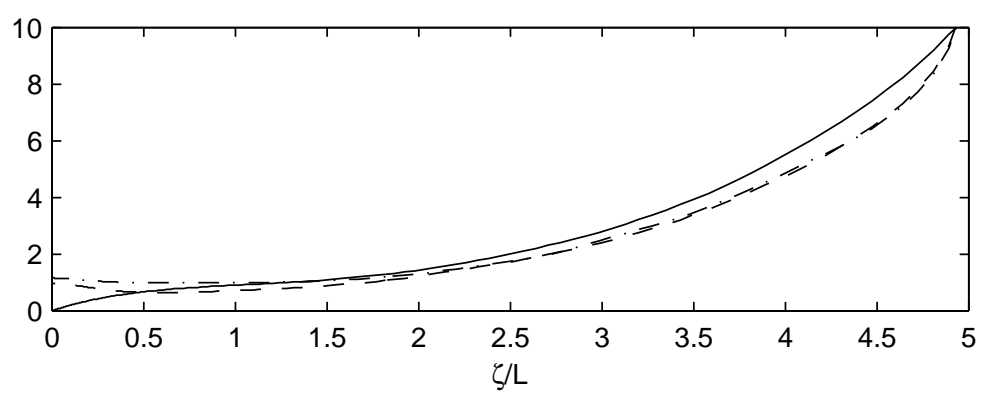

b)

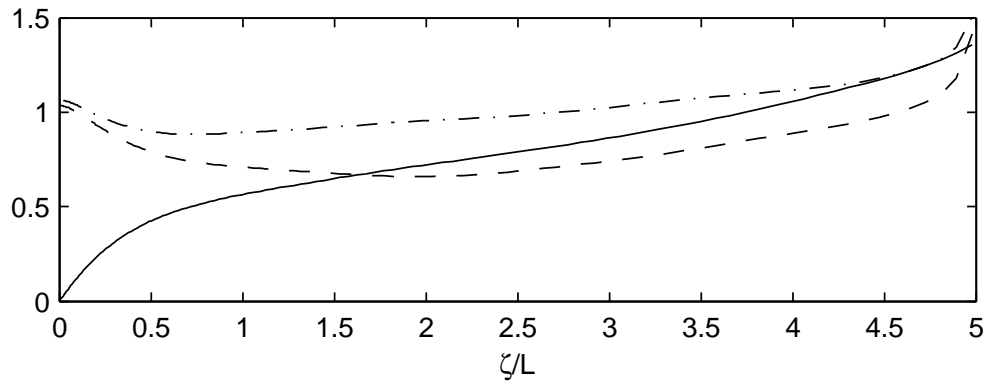

FIGURE 5. Decay of turbulent velocity fluctuations with increasing distance away from the turbulence generation source which is located at the bottom $(\zeta / L=5)$ of the domain. The velocities are normalized with $\sqrt{k_{\nu}}$ where $k_{\nu}$ is the turbulent kinetic energy at one- $L_{\nu}$ below the surface $\left(L_{\nu}\right.$ is the thickness of the viscous sublayer defined below): $-\cdot-u_{r m s} / \sqrt{k_{\nu}},---$ $v_{r m s} / \sqrt{k_{\nu}},-w_{r m s} / \sqrt{k_{\nu}}$. a)from case GS80 and b) case GS500.

turnover times due to the high numerical cost of the calculation of the scalar transfer on the refined mesh.

The distribution of the turbulent fluctuations and the turbulent kinetic energy $(k)$ in the near-surface region are shown in Figures 6a and b for the cases GS80 and GS500, respectively. As a consequence of the rigid lid assumption, the flow looses its isotropy when approaching the interface. The vertical velocity decreases rapidly to zero while the non-restricted horizontal components slightly increase. In the experiments of Herlina \& Jirka (2008) (used as one of our reference experiments) the horizontal turbulent fluctuations were found to decrease near the surface, most probably due to the presence of tracer particles at the surface. This dampening of the turbulent fluctuations near contaminated surfaces was, for instance, also observed in the laboratory experiments of McKenna \& McGillis (2004b) and in recent low Sc DNS simulations of Khakpour et al. (2011) who employed a boundary condition at the surface which accounts for the relation of surface tension to surfactant concentration.

Also shown in Figure 6 is the typical increase of $k$ near the free surface $(\zeta / L \leqslant 0.5)$ - corresponding to the sudden decrease in $w_{r m s}$ and the slight increase of $u_{r m s}$ and $v_{r m s}$ mentioned above - as has been found previously in experiments (e.g. Komori et al. 1982) and numerical simulations (e.g. Calmet \& Magnaudet 2003; Walker et al. 1996). In Figure $6, k$ is normalized with $k_{\nu}$ which is the turbulent kinetic energy at one- $L_{\nu}$ below the surface $\left(L_{\nu}\right.$ is the thickness of the viscous sublayer as defined below). The minimum values of $k / k_{\nu}$ were found to be 0.88 and 0.79 in cases GS80 and GS500, respectively, 
a)

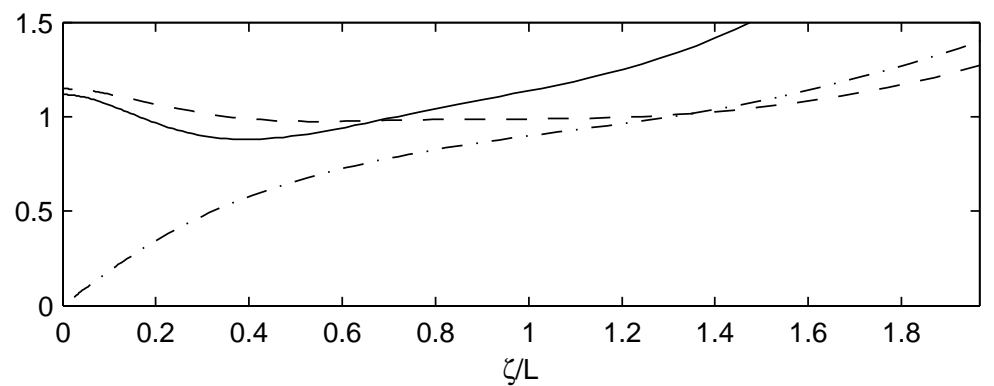

b)

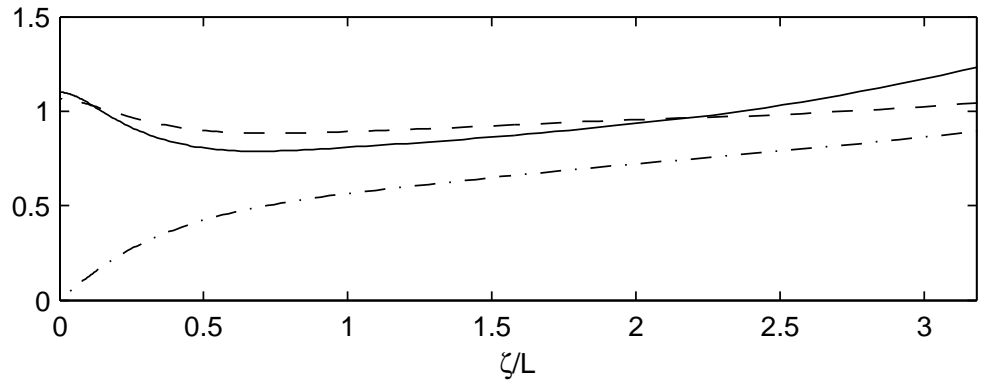

FIgURE 6 . Distribution of the velocity fluctuations $u_{r m s} / \sqrt{k_{\nu}}$ and $w_{r m s} / \sqrt{k_{\nu}}$ and the turbulent kinetic energy $k / k_{\nu}$ near the surface region: $-k / k_{\nu},---u_{r m s},-.-. w_{r m s}$ (a) case GS80 and (b) case GS500.

which are comparable with the value $k_{m i n} / k_{\nu}=0.865$ predicted by Hunt \& Graham (1978) and $k_{\min } / k_{\nu} \approx 0.84$ obtained in the open-channel flow computations of Calmet \& Magnaudet (2003).

In Figure $7 \mathrm{a}$, the variation of the local integral length scale $L_{11}$ with depth is displayed. The evaluation of $L_{11}$ was performed by integrating the two-point longitudinal velocity correlation function $\left(R_{11}\right)$,

$$
L_{11}=\int_{0}^{\infty} R_{11}\left(r_{1}\right) d r_{1},
$$

where $r$ denotes the distance between the points. Because the dissipation tends to be small at large length scales, the turbulence-decay in the large integral length scale case GS500 was found to be smaller than in GS80 (see Figures 5a and b). As observed in grid-stirred experiments, the size of the eddy structures grows with distance from the turbulence source at the bottom. Near the interface $L_{11}$ reaches a maximum value which is related to the size of the largest eddy resolved in our domain. This maximum value is taken as the characteristic integral length scale of the flow field $\left(L_{\infty}\right)$.

As evidenced in Figure 5, the thickness of the so called surface influenced layer (Brumley \& Jirka 1988) corresponding to the depth over which the flow becomes anisotropic or, in other words, over which the surface blockage is felt matches $L_{\infty}$. The thickness $L_{\nu}$ of the viscous sublayer is defined by the distance over which $\partial u_{\mathrm{rms}} / \partial z$ decreases from its maximum value to zero as forced by the rigid lid assumption near the surface (Figure 7b). $L_{\nu}$ in our DNSs was found to be of the order of $L_{\infty} R_{T}^{-1 / 2}$ (i.e. $L_{\nu}=0.7 L_{\infty} R_{T}^{-1 / 2}$ in GS80 and GS200; and $L_{\nu}=L_{\infty} R_{T}^{-1 / 2}$ in GS500) which is in good agreement with 

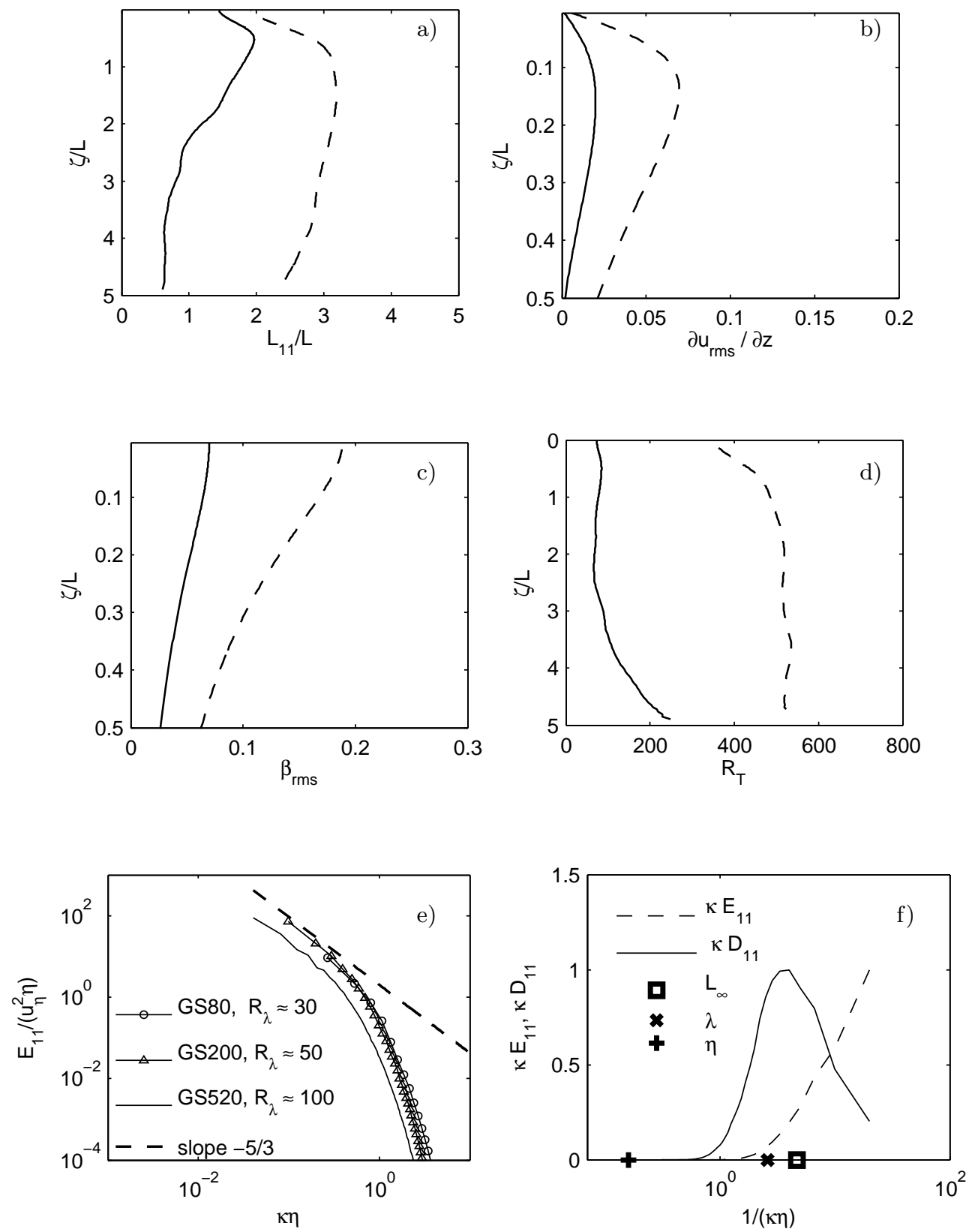

FiguRE 7 . Flow characteristics over depth where $\zeta / L$ denotes the distance from the interface. In a) to d): — and - - - represent the cases with the lowest (GS80) and highest (GS500) turbulent Reynolds numbers, respectively. a) Local integral length scale $L_{11}$ estimated based on the $u$-fluctuations longitudinal correlation function (1-sided). b) Profiles of $\partial u_{r m s} / \partial z$. c) Profiles of $\beta_{r m s}$ (rms of surface divergence $\beta$ ). d) Local turbulent Reynolds number $R_{T}$. e) 1-velocity energy spectra: time and space averaged at a selected $\zeta / L$ plane. f) Premultiplied energy and dissipation spectra from case GS200 (scaled with their maximum value). 
the prediction of Hunt (1984). While $\partial u_{\mathrm{rms}} / \partial z$ decreases towards the surface within $L_{\nu}$, at clean surfaces the rms of the surface divergence $\beta_{\mathrm{rms}}(\beta=-\partial w / \partial z)$ keeps increasing (Figure 7c). The profiles also indicate the thickness $\eta$ of the Kolmogorov sublayer where the vertical velocity decreases linearly with $\zeta / L$ (see e.g. Pope 2000). The computed predictions of $\eta$, based on the relation $\eta=\left(\nu^{3} / \epsilon\right)^{1 / 4}$, scale approximately with $\eta=2 L_{\infty} R_{T}^{-3 / 4}$ (Brumley \& Jirka 1988).

Based on the local $u_{\mathrm{rms}}$ and the local correlation length scale $L_{11}$, the variation of the turbulent Reynolds number $R_{T}\left(R_{T}=u_{\mathrm{rms}} 2 L_{11} / \nu\right)$ with $\zeta / L$ (Figure $7 \mathrm{~d}$ ) was computed. In the grid-stirred experiments, at a sufficient distance away from the turbulence source, the turbulent fluctuations $u_{\text {rms }}$ were observed to decay linearly with $z$ while $L_{\infty}$ increased linearly with $z$ (Herlina \& Jirka 2008) resulting in a constant $R_{T}$. The same behaviour was found in the present DNS: $R_{T}$ stayed almost constant at values of 84 and 507 for GS80 and GS500, respectively. When determining the global $R_{T}$ of the system, for consistency reasons the velocity scale $u_{\infty}$ was identified with the value of $u_{\mathrm{rms}}$ at the location of maximum $L_{11}$. Based on these two quantities and the kinematic viscosity of the fluid turbulent Reynolds numbers of approximately 84, 195 and 507 in GS80, GS200 and GS500, respectively, were obtained. According to Theofanous (1984), these Reynolds numbers are all in the low to moderate regimes. The selection of $u_{\infty}$ and $L_{\infty}$ as the characteristic velocity and length scales was found to be appropriate as the resulting $R_{T}$-s lie within the bulk region where the local $R_{T}$-s remain constant.

Besides the turbulent Reynolds number $R_{T}$, another useful measure of the turbulence level is the Taylor Reynolds number $\left(R_{\lambda}=u_{\mathrm{rms}} \lambda / \nu\right)$, where $\lambda$ is the Taylor microscale that can be obtained from the relation

$$
\overline{\left(\frac{\partial u}{\partial x}\right)^{2}}=\frac{2 u_{\mathrm{rms}}^{2}}{\lambda^{2}}
$$

The mean Taylor Reynolds numbers estimated some distance away from the surface (at $\zeta / L=0.7$ in GS80 and GS500 and at $\zeta / L=0.46$ in GS200) were between 30 and 100. Although $R_{\lambda}$ was in the lower range, we could still observe the existence of an inertial range as evidenced in the computed spectrum (Figure 7e). In Figure $7 \mathrm{f}$ the premultiplied energy and dissipation spectra are shown for GS200 $\left(R_{\lambda}=50\right)$. As expected for low $R_{\lambda}$ number flows, the energy and dissipation spectra overlap. The symbols in Figure $7 \mathrm{f}$ indicate the sizes of the integral, Taylor and Kolmogorov length scales. Because of the need to fully resolve the diffusive sublayer, our mesh size was chosen to be finer than the Kolmogorov length scale (see Table 2).

\section{Statistical properties of turbulent scalar transport}

The interfacial mass transfer in the present system is affected by the molecular diffusivity ( $S c$ dependency) of the solvent itself and the level of the turbulence rising up from below $\left(R_{T}\right.$ dependency). As described in Section 2 , the mass transfer was simulated at three different $R_{T}$. At each $R_{T}$ mass transfer was calculated for five to six $S c$ numbers. The cases with $S c=2$ to 32 were solved simultaneously in single runs such that the mass transfer could be investigated using exactly the same background turbulent flow-field allowing an unbiased (direct) $S c$ parametric study over the whole simulation time. As mentioned previously in Section 2, for the $S c=500$ cases GS80R5 and GS200R5 the same initial flow-field was used as in the lower $S c$ cases $(S c=2$ to 32$)$ so that a direct parametric study of the Schmidt number could be made from $t / T=0$ up to (at least) 

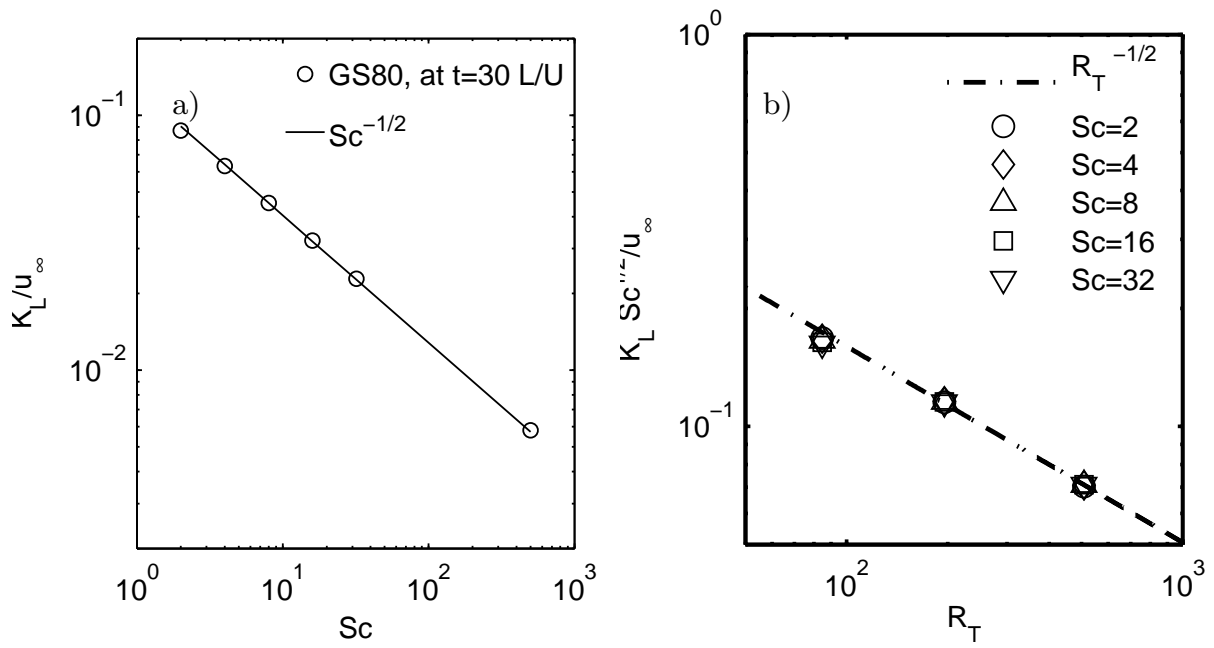

FiguRE 8. Variation of the total transfer velocity $K_{L}$ with (a) Schmidt number $S c$ and (b) turbulent Reynolds number $R_{T}$.

the time when the evolution of the turbulent flow field started to differ from that in the lower $S c$ cases.

A detailed discussion of the instantaneous flow structures that enhance the scalar transport can be found in the later sections. In this section the mean properties of the scalar transport, in particular the influence of $S c$ and $R_{T}$, will be discussed.

\subsection{Transfer velocity}

The instantaneous gas flux $j$ at the interface $i$ is completely dominated by diffusion and can be written as

$$
j_{i}=\left.D \frac{\partial c}{\partial z}\right|_{i}
$$

The averaged transfer velocity $K_{L}$ can subsequently be determined using the relation

$$
\bar{j}=K_{L}\left(c_{s}-c_{b}\right)=\left.D \frac{\partial \bar{c}}{\partial z}\right|_{i}
$$

where $c_{s}$ and $c_{b}$ are the saturation concentrations at the interface and in the bulk, respectively. Figures 8a and b show the influence of the Schmidt $S c$ and turbulent Reynolds numbers $R_{T}$, respectively, on the transfer velocity $K_{L}$. Figure 8a shows that the overall total mass flux is related to the Schmidt number by $S c^{-1 / 2}$ which is in agreement with the theory for freely moving interface boundary conditions. Though previous DNSs have shown that the same dependency holds for low $S c$ numbers (less than 8), as far as the authors are aware this is the first DNS that shows that it also holds for $S c$ numbers up to 500. The present results indicate that the usage of temperature (heat) ( $S c \sim \operatorname{Pr}=7$, where $\mathrm{Pr}$ is the Prandtl number) as a tracer for the measurements of the mean gas transfer velocity $K_{L}$ might be possible provided that the temperature behaves approximately like a passive scalar and the effect of evaporation (latent heat transfer) on the temperature field is negligible. In additon, it should be kept in mind that the power rate dependency of $-1 / 2$ only applies at clean surfaces where shear stresses are approximately 


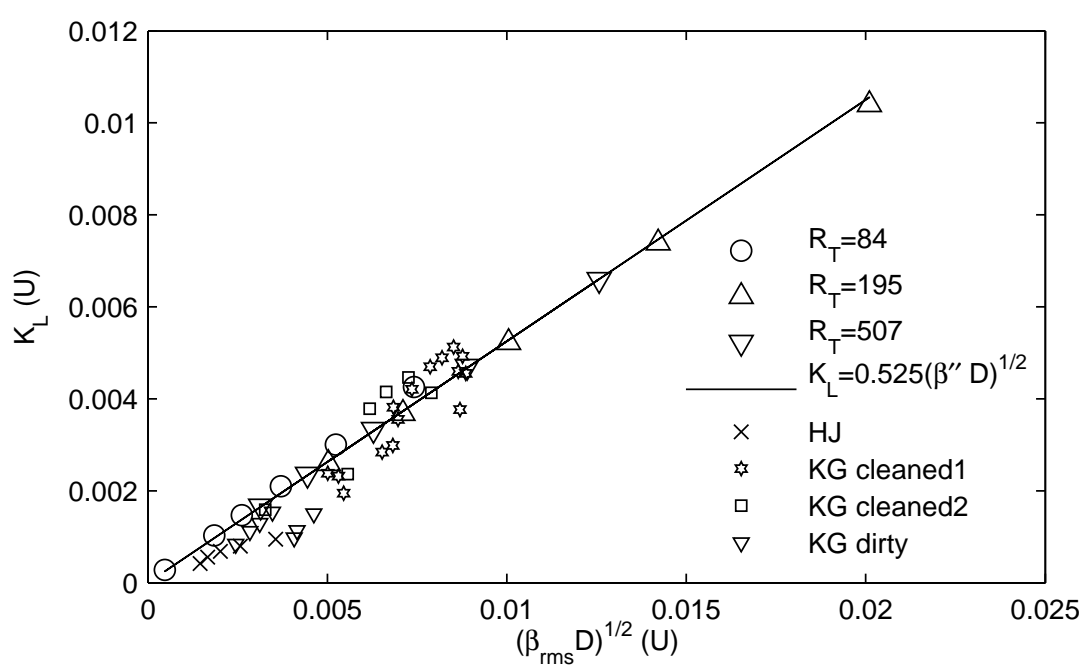

FiguRE 9. Estimation of $K_{L}$ using the surface divergence model of McCready et al. (1986). The present numerical data are in good agreement with the surface divergence model with a constant of proportionality of 0.525 . The symbols indicated with $\mathrm{HJ}$ and $\mathrm{KG}$ are experimental data from Herlina \& Jirka (2008) and McKenna \& McGillis (2004a), respectively.

zero. However, when the interface is not clean, for example when it is covered by surfactans, greater tangential stresses occur and the turbulence fluctuations are damped. At very contaminated surfaces, the description of the mass flux would be similar to the theoretical relations of solid-liquid boundary conditions $\left(S c^{-2 / 3}\right.$, see Jähne \& Haussecker 1998).

In Figure 8b, the variation of the mean $K_{L}$ with $R_{T}$ is shown. Within the present range of $R_{T}$, the DNS data show that $K_{L} / u_{\infty}$ scales with $R_{T}{ }^{-1 / 2}$. Using the above result which showed that $K_{L} \propto S c^{-1 / 2}$, we can conclude that for our range of Schmidt and turbulent Reynolds numbers $K_{L}$ can be written as

$$
K_{L}=a u_{\infty} R_{T}^{-1 / 2} S c^{-1 / 2}
$$

which is similar to the large-eddy model proposed by Fortescue \& Pearson (1967). The constant $a$ in the present simulations is found to be about 1.6. In the grid-stirred experiments of Herlina \& Jirka (2008) $K_{L}$ (measured in the bulk) was found to generally follow $R_{T}^{-1 / 4}$. However, it should be noted that compared to the present DNS-s their measurements spanned also to higher $R_{T}$, where for $R_{T}>500$ Theofanous (1984) suggested that the small-eddies scaling as proposed by Lamont \& Scott (1970) and Banerjee et al. (1968) would be more appropriate $\left(K_{L}=c u_{\infty} R_{T}^{-1 / 4} S c^{-1 / 2}\right)$.

In addition, as mentioned above, $K_{L}$ is sensitive to the surface condition which is reflected in the surface divergence $\beta$. Laboratory measurements have indeed shown $\beta_{\text {rms }}$ to be a good measure for mass transfer as it implicitly takes the surface conditions into account (Turney \& Banerjee 2013). Figure 9 shows the dependency of $K_{L}$ on the surface divergence supporting the surface divergence model of McCready et al. (1986), $K_{L} \propto$ $\sqrt{\beta_{\mathrm{rms}} D}$. It can be seen that our numerical results predict that $K_{L}=0.525 \sqrt{\beta_{\mathrm{rms}} D}$ which matches the trend of the experimental results in grid-stirred tanks performed by Herlina \& Jirka (2008); McKenna \& McGillis (2004a). The advantage of the present numerical 
simulation is that surface contamination is not an issue so that unbiased investigations of the effect of $S c$ and the turbulence levels (here described in terms of the turbulent Reynolds number $R_{T}$ ) can be performed.

\subsection{Boundary layer}

The instantaneous snapshots in Figure 13 present a visual impression of the effect of impinging turbulence on the diffusive boundary layer at $S c=2$ and $S c=32$. As shown in Figure 13b, a decreased diffusivity at the higher $S c$ case $(S c=32)$ causes the concentration (or diffusive) boundary layer to become thinner. From below the boundary layer is constantly disturbed by the turbulent flow: upwelling motions suppress the thickness of the layer while downwelling motions locally thicken the layer. In the mean, the presence of turbulence diffusing upwards from below suppresses the diffusive boundary layer leading to a steeper concentration gradient which increases the diffusivity controlled transfer rate at the surface. Obviously, the thickness $\delta$ depends on both $R_{T}$ and $S c$. This makes $\delta$ an appropriate length scale when analysing the scalar properties as $\delta$ represents a mixed length scale taking into account both the kinematic viscosity of the fluid and the molecular diffusivity of the transported scalar (see also the discussion by Magnaudet \& Calmet (2006)).

Various definitions to quantify $\delta$ have been used in the literature. They mostly depend on the available or resolvable data. For instance, $\delta$ could be defined as the depth where the peak of the concentration fluctuation occurs $\left(\delta_{c f}\right)$ or -due to the concentration profile being approximately exponential- as $\delta_{e}$ which corresponds to the distance from the surface where the normalized concentration, given by

$$
\left(c-c_{b}\right) /\left(c_{s}-c_{b}\right),
$$

reaches a value of $1 / e$ with $e$ being the Euler number $(e=2.71828182 \ldots)$ and $c_{b}$ is the mean bulk concentration at a certain reference depth. (If not mentioned otherwise, we take $c_{b}$ as the mean value of $c$ at a distance of $L_{\infty}$ from the surface.) Often, the boundary layer thickness is quantified by a fictious boundary layer thickness $\delta_{i}$ defined by

$$
\delta_{i}=D\left(c_{s}-c_{b}\right) / \bar{j}
$$

With the latter definition, the overall mass transfer across the interface, as discussed by Jähne \& Haussecker (1998), can be simply described using the transfer velocity $K_{L}$, the concentration boundary layer thickness $\delta_{i}$ and the time interval $t_{i}$ as scaling parameters owing to the fact that they are directly related to each other through the molecular diffusion coefficient $D$, i.e. $K_{L}=D / \delta_{i}$ and $t_{i}=\delta_{i} / K_{L}=D / K_{L}^{2}$. The relation $K_{L}=$ $D / \delta_{i}$ should not be misinterpreted as $K_{L}$ being linearly dependent on $D$, such as in the assumption of the classical film model of Lewis \& Whitman. It will be shown below that for mass transfer at clean surfaces, $\delta_{i}$ is a function of $(\nu / D)^{-1 / 2}$ so that $K_{L}$ is proportional to $\sqrt{D}$ as confirmed by laboratory experiments and predicted by the surface renewal model of Danckwerts (1951).

Figure 10a shows the concentration boundary layer thickness $\delta$ from GS200, where $\delta$ is estimated using the various definitions described above. All three definitions of $\delta$ scale with $S c^{-1 / 2}$ and only a slight variation between the three thicknesses is observed, i.e. $\delta_{e} \leqslant \delta_{i} \leqslant \delta_{c f}$ so that all three definitions are equally useful. Also plotted in Figure 10a are the different hydrodynamic layers arising near the free surface, which are calculated using the scaling laws as described in Brumley \& Jirka (1988). These include the surface influenced layer $L_{\infty}$ used to normalize the depth $\zeta$, the viscous sublayer $L_{\nu}=L_{\infty} R_{T}^{-1 / 2}$, the Kolmogorov sublayer $\eta=2 L_{\infty} R_{T}^{-3 / 4}$ and the two concentration (diffusive) sublayers 

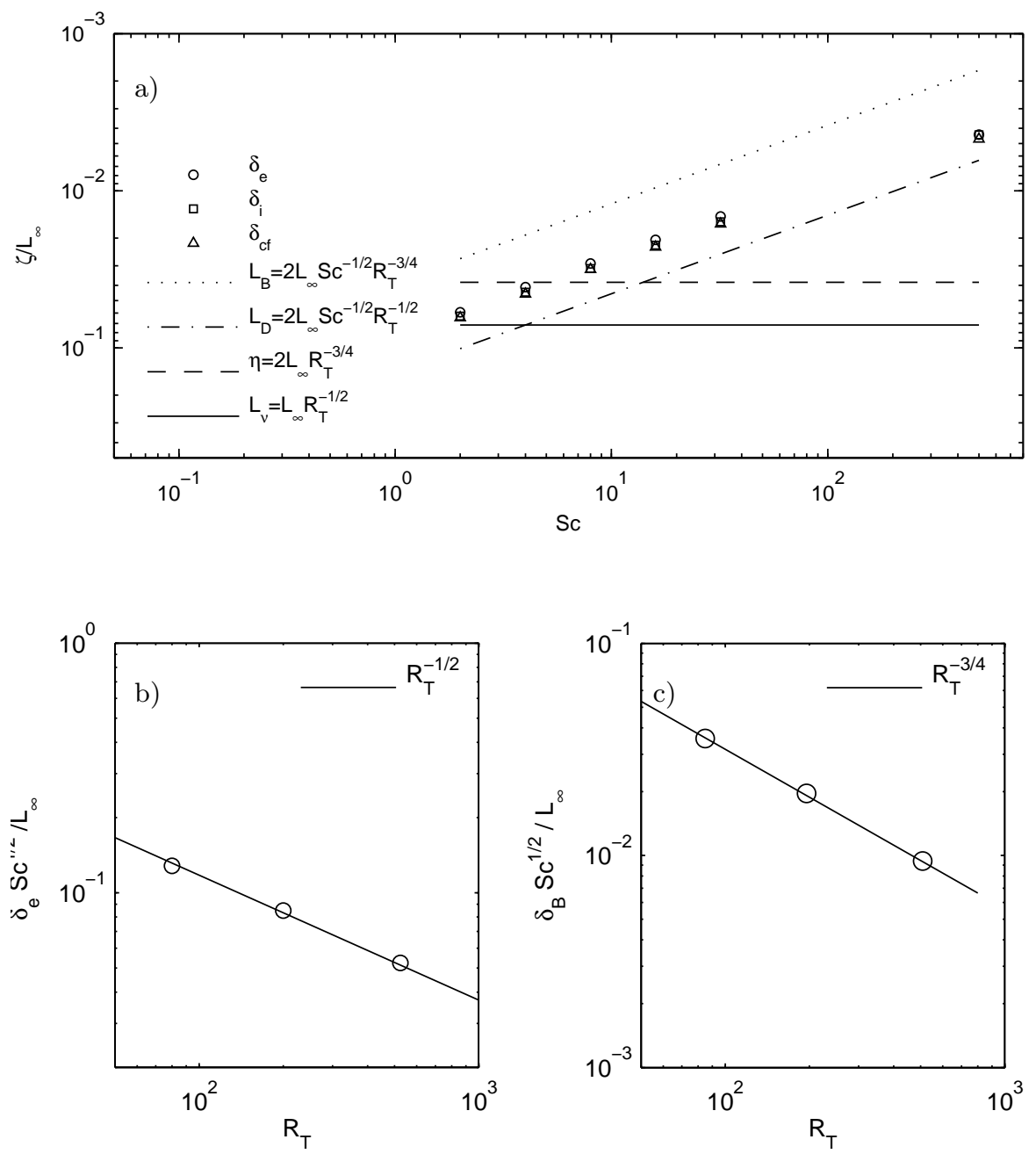

FiguRE 10. (a) Variation of boundary layer thickness $\delta$ at $R_{T}=195$ (GS200 and GS200R5) with Schmidt number $S c$ and comparison to the different sublayer thicknesses, (b) Variation of observed $\delta_{e}$ with $R_{T}$ and (c) Variation of observed $\delta_{B}$ (layer in which $c$ evolves linearly) with $R_{T}$.

arising from the largest and the smallest eddies present in the system. The first three sublayers have been discussed in Section 4. The latter two sublayers are usually referred to as the outer diffusive sublayer $L_{D}=2 L_{\infty} S c^{-1 / 2} R_{T}^{-1 / 2}$ and the Batchelor sublayer $L_{B}=2 L_{\infty} S c^{-1 / 2} R_{T}^{-3 / 4}$, respectively. For the case $R_{T}=195$ (Figure 10a), the boundary layer thickness $\delta$ for all $S c$ resides within the viscous sublayer thickness $L_{\nu}$. At $S c \geqslant 8, \delta$ is even smaller than the Kolmogorov length scale. For the lower $R_{T}$ cases an exception was found for $S c \leqslant 2$ where $\delta$ was located outside the observed viscous sublayer. In all runs, the boundary layer thickness was found to scale with $S c^{-1 / 2}$.

Subsequently the effect of $R_{T}$ on the thickness of the diffusive sublayers was examined. Figure $10 \mathrm{~b}$ shows that in the present range of simulated $R_{T}$ the thickness of the 
concentration boundary layer $\delta_{e}$ varies with $R_{T}^{-1 / 2}$. The thickness $\delta$ can thus be associated with the outer diffusive sublayer thickness $L_{D}$. In the present DNS-s, it was found that $\delta_{e} \approx 0.58 L_{D}$. In the grid stirred experiments by Herlina \& Jirka $(2008) \delta_{e}$ was $\approx 1.9 L_{D}$. The increased thickness of the diffusive boundary layer in the experiments is likely to be related to the contaminated surface which increases the damping of the velocity fluctuations.

As mentioned in the previous section, our vertical grid spacing was sufficiently fine to resolve both the Kolmogorov $\eta$ and the Batchelor $L_{B}$ sublayer thicknesses. Figure 10c shows the variation of the thickness $\delta_{B}$ with $R_{T}$, where $\delta_{B}$ is the distance from the surface over which the concentration profile evolves linearly with $\zeta$ (and, hence, diffusion completely dominates). It was found that $\delta_{B} \approx L_{\infty} S c^{-1 / 2} R_{T}^{-3 / 4}$, corresponding to $50 \%$ of the estimated inner diffusive sublayer thickness $L_{B}$ as suggested by Brumley \& Jirka (1988).

\subsection{Mean and fluctuation concentration profile}

Figure 11a presents the mean normalized concentration distribution in the vertical direction from GS500. The distance from the surface, denoted by $\zeta$, is normalized with $\delta_{e}$, while the concentration is given by Equation 5.4. The profiles obtained at various $S c$ numbers nearly collapse onto the same curve, illustrating that the boundary layer thickness $\delta_{e}$ can be used as a representative length scale when analysing the concentration profile. In the deeper regions, a slight deviation between curves can be observed. The deviation was found to depend on the reference depth at which $c_{b}$ is determined. As the downward transport of dissolved gases at higher $S c$ is reduced, the quasi-steady state condition at deeper reference points is achieved later in time than in the lower $S c$ cases.

For various $R_{T}$, the mean concentration profiles at $S c=32$ are shown in Figure 11b. The profiles almost collapse onto the same curve which is in agreement with the trend found by Herlina \& Jirka (2008). As $R_{T}$ increases the spatial drop-off (the rate of concentration decay) slightly decreases, which might be an indication that apart from $\delta_{e}$ (associated with the integral length scales) other, smaller, scales could also be important. Such multiscale processes can be observed in the instantaneous snapshots discussed in the next section.

The normalized root mean squared concentration profiles $\left(c_{\mathrm{rms}}\right)$ are presented in Figures $11 \mathrm{c}$ and $\mathrm{d}$. The rms of the fluctuations increases from 0 at the interface and reaches a maximum value near the boundary layer edge. For all runs the peak value was found to be between 0.25 and 0.3 , which was higher than in the experiments by Herlina \& Jirka (2008), but largely in agreement with the values reported by various other researchers, which vary between 0.1 and 0.3 . The large variation measured in the laboratory might be due to difficulties in maintaining the exact same surface conditions as well as limitations of measurement techniques when it comes to measuring minute fluctuations within the boundary. It should also be noted that it is nearly impossible to maintain a perfectly clean surface in the laboratory. Most of the time, the water surface had a certain degree of contamination, for instance due to dust particles. Khakpour et al. (2011) showed a decrease of the concentration fluctuations near contaminated surfaces when compared to clean surfaces. This is related to the increased damping of the turbulent velocity fluctuations near contaminated surfaces.

\subsection{Turbulent Mass flux}

To determine the total mass flux $\bar{j}$ it is necessary to know both the turbulent and diffusive mass fluxes (see (1.2)). Measurements of the turbulent mass flux $c^{\prime} w^{\prime}$ using the eddycorrelation method are difficult and the first attempt to measure $c^{\prime} w^{\prime}$ in grid-stirred 

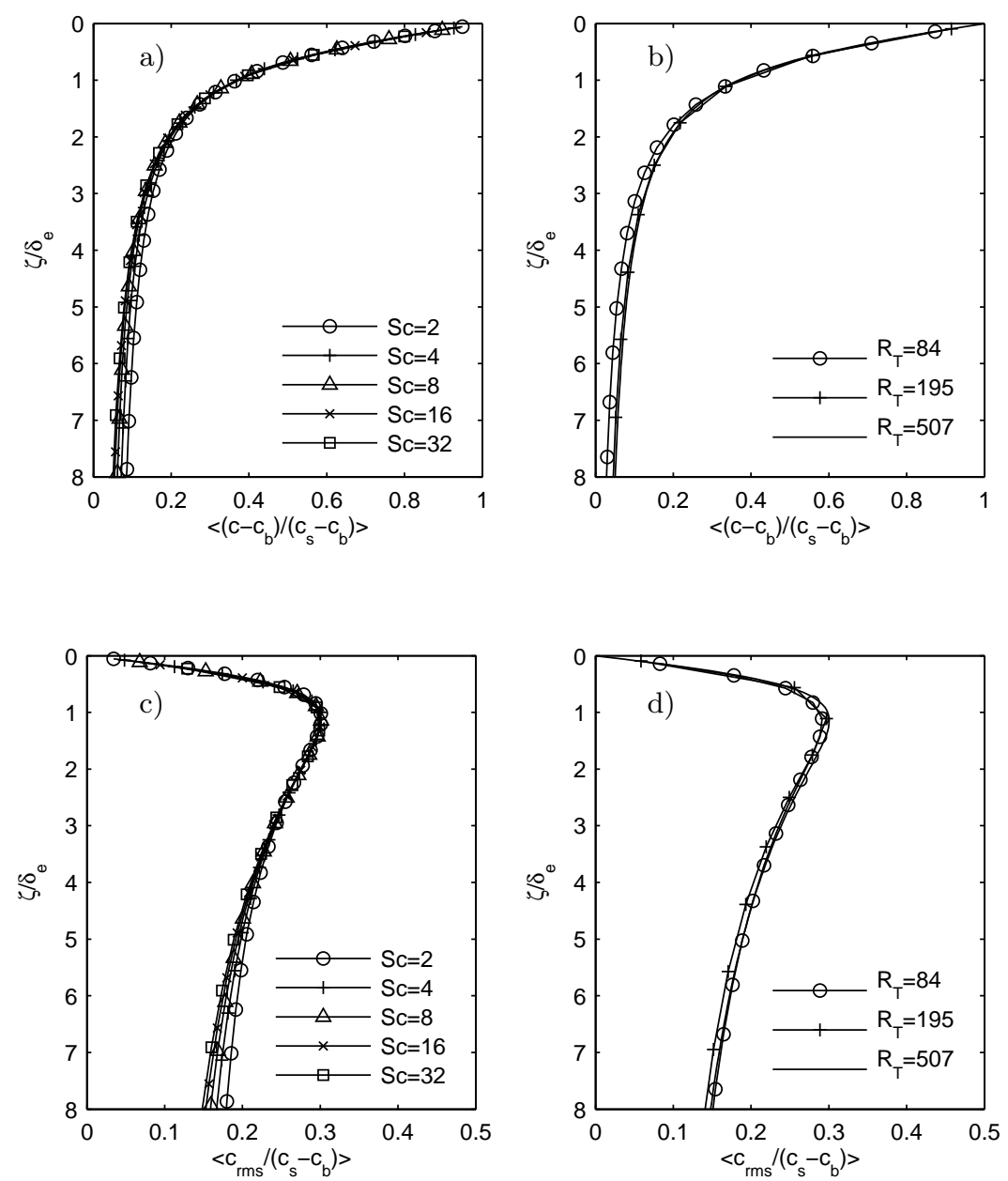

FIGURE 11. Mean and fluctuation concentration profiles. a) Influence of $S c$ on $c$ data from $\operatorname{GS} 500\left(R_{T}=507\right)$, b) Influence of $R_{T}$ at $\left.\mathrm{Sc}=32 . \mathrm{c}\right)$ Variation of $c_{\mathrm{rms}}$ from run $\operatorname{GS} 500\left(R_{T}=507\right)$, b) Comparison between the three cases for different turbulent Reynolds numbers at $S c=32$.

experiments by Chu \& Jirka (1992) was inconclusive. Herlina and Jirka's (2008) data (Figure 12c) showed a typical increase of the turbulent mass fluxes $\overline{c^{\prime} w^{\prime}}$ with increasing distance from the surface but this observation was limited to the region between $\zeta / \delta=0$ and about $2-3$.

In Figure 12a the variation of the time-space averaged molecular $(D \partial \bar{c} / \partial z)$ and turbulent $\left(\overline{c^{\prime} w^{\prime}}\right)$ mass fluxes with depth carried out for $S c=2$ to 32 is presented for GS200. The ordinate shows the depth normalized with the thickness of the viscous layer. These figures clearly illustrate the effect of the Schmidt number on the mass fluxes. At low Schmidt numbers, the region where molecular diffusion dominates is relatively thick. At $S c=32$, however, the contribution of the turbulent fluxes is already of order $D \partial \bar{c} / \partial z$ almost immediately below the surface.

The normalized mass flux profiles for all $R_{T}$ are shown in Figure 12b. At the surface, the turbulent mass fluxes are zero as any turbulent transport vanishes in the immediate 

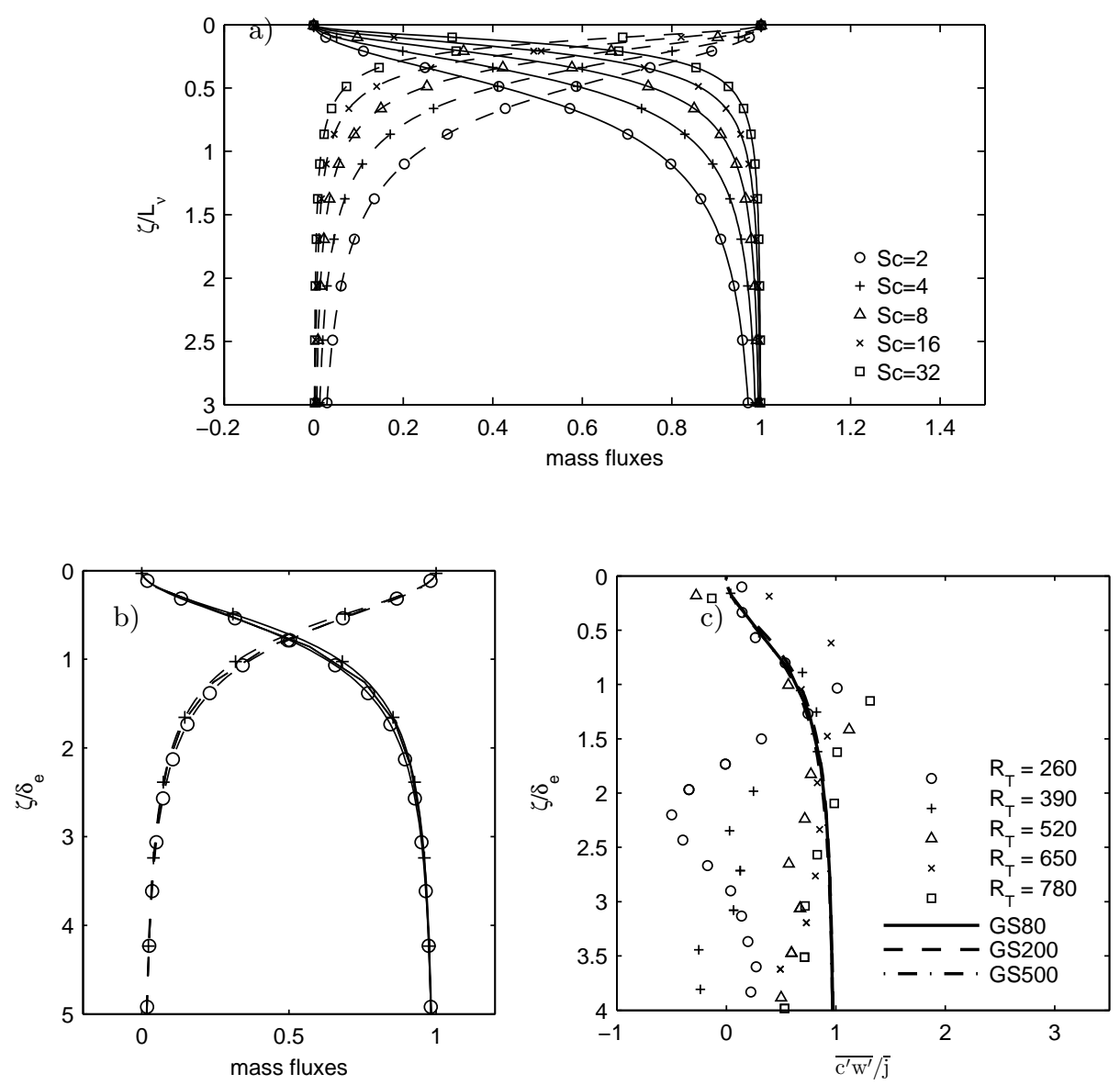

Figure 12. Variation of mass fluxes with depth. The mass fluxes are normalized with the total flux $\left(D \partial \bar{c} / \partial z+\overline{c^{\prime} w^{\prime}}\right)$. a) Molecular $D \partial \bar{c} / \partial z$ (dashed lines) and turbulent $\overline{c^{\prime} w^{\prime}}$ (solid lines) mass fluxes plotted against $\zeta / L_{\nu}$ at various $S c$ numbers. b) Molecular (dashed lines) and turbulent (solid lines) mass fluxes $(S c=32)$ at $R_{T}=84,195$ and 507 (legend for the symbols is the same as in Figure 11d). c) Mean profiles of $c^{\prime} w^{\prime}$ measured in the grid-stirred tank experiments of Herlina \& Jirka (2008).

vicinity of the water surface. In this region the molecular diffusion contribution to the transfer mechanism is maximum but reduces quickly to $50 \%$ at around $0.75 \delta_{e}$. After this point, the importance of the turbulent mass flux increases rapidly and beyond $z \geqslant 3 \delta_{e}$ it becomes almost completely dominant.

As shown in Figure 12c, for $0<\zeta / \delta_{e} \leqslant 2.5$ a very good agreement between the numerical results and the experimental data from Herlina and Jirka (2008) is obtained. For larger $\zeta / \delta_{e}$ the experimental $\overline{c^{\prime} w^{\prime}}$ profiles tend to decrease to zero. This decrease was unexpected as both the numerical and experimental data were gathered in the quasistatic regime. The most likely reason for this discrepancy with both the present numerical results and the theory is that the laser intensity of the LIF setup was insufficient to detect the minute concentration fluctuations in the deeper bulk region. Figures $12 \mathrm{a}$ and b, hence, emphasize the major advantage of DNS compared to even advanced experiments which 
is that DNS allows us to elucidate the distribution of $D \partial \bar{c} / \partial z$ and turbulent $\overline{c^{\prime} w^{\prime}}$ within the diffusive sublayer down to the deeper bulk region. The present results show that experimental measurements and DNS results complement each other well in achieving a better understanding of the gas transfer mechanisms.

\section{Flow and scalar structures near the surface}

Figure 13 compares contours of the scalar distributions for $S c=2$ and 32 in the crosssection at $x / L=2.5$ from simulation GS80 $\left(R_{T}=84\right)$. The superposed velocity vectors correspond to a snapshot of the turbulent flow-field that drives the scalar transfer and is the same for both $S c$ numbers. The 2D visualizations show the interplay between the upand downwelling motions and the scalar transport. In general, similar scalar patterns or structures are seen. The major differences are that in the higher Schmidt number case $(S c=32)$ the concentration boundary layer is much thinner, the structures are finer and the concentration gradients steeper.

In Figures 14a and c, two snapshots of the concentration field captured in the laboratory grid-stirred experiments (using the Laser Induced Fluorescence (LIF) technique) at $R_{T}=260$ and $R_{T}=520$, respectively, are presented for qualitative comparison to the present numerical results at $R_{T}=195$ (GS200) and $R_{T}=507$ (GS500). Similar concentration distributions as found in the experiments were observed (Figures 14b and d). In Figures $14 \mathrm{c}$ and $\mathrm{d}$ it can be seen that the transport mechanisms of the highly concentrated mass from the surface into the bulk are similar despite the huge differences between the Schmidt numbers in the numerical simulation $(S c=32)$ and the experiment $(S c \approx 500)$. It is interesting to note that (for the $S c=500$ case) in the deeper bulk region fine tail structures move in and out of the $2 \mathrm{D}$ vertical observation plane (Figures 14a and b). Similar tail structures (Schlieren) were also recorded in the LIF images of Herlina \& Jirka (2004). The present DNS results hence corroborate that the Schlieren structures seen in the experiments are truly physical and not just optical artefacts.

Compared to the 2D LIF measurements, in the DNS instantaneous 3D spatial information of the flow-field and the gas distribution could be obtained allowing a more detailed examination of the physical processes involved. For instance, Figure 15a shows a contour plot of the divergence in the horizontal section immediately below the surface illustrating upwelling and downwelling action of turbulent motions impinging on the surface from below. It can be seen that upwellings can be very strong locally but that these occurences are quite rare. These strong upwellings are located approximately in the middle of the large cells, though the structures are not always symmetric. Once the fluid is very close to the air/water interface, it starts to flow radially outward, almost parallel to the interface with only a very small vertical component $w$ (as can be seen in the PDF in Figure $15 \mathrm{~b}), w$ very often is slightly negative - as the initial upwelling may cause some overshoot. The strong downwellings occur in very narrow regions that separate cells formed by the upwelling and subsequent radial motion of the fluid. This is similar to the flow of a vertical jet impinging on a horizontal surface, where the stagnation point would be approximately in the middle of the divergence area.

In Figure 15b, the probability density function (PDF) of surface divergence occurrences stored every 0.1 time-units during 4 eddy turn-over times of simulation GS200 is displayed. All runs were found to have a similar PDF. The PDF shows that most occurences by far are low velocity up- and downwelling events which more or less correspond to (almost) wall-parallel flow (as supported by the predominant blue to green colour scaling in Figure 15a). The direct connection of the flow-field structures to the scalar distribution near the surface is discussed below. 
a)

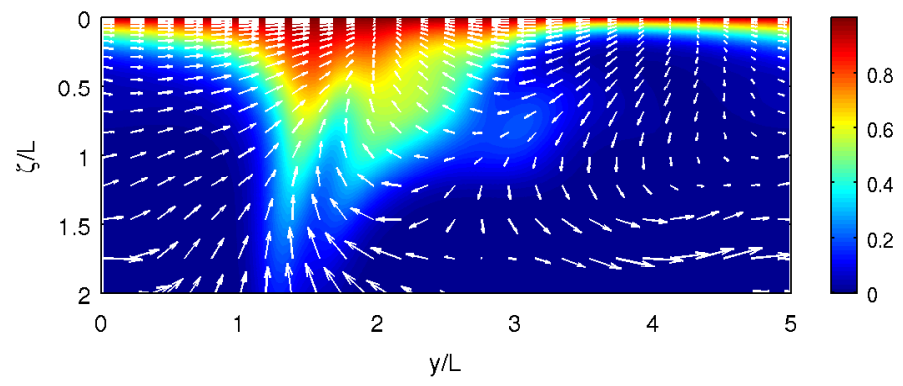

b)

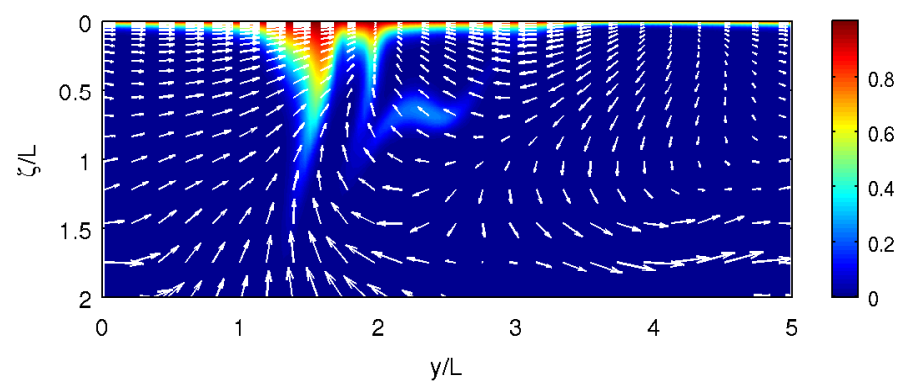

FIgURE 13. Visualization of scalar transport obtained from Run $\operatorname{GS} 80\left(R_{T}=84\right)$ for the cases (a) $S c=2$ and (b) $S c=32$. The thickness of the boundary layer reduces with increasing Schmidt number.
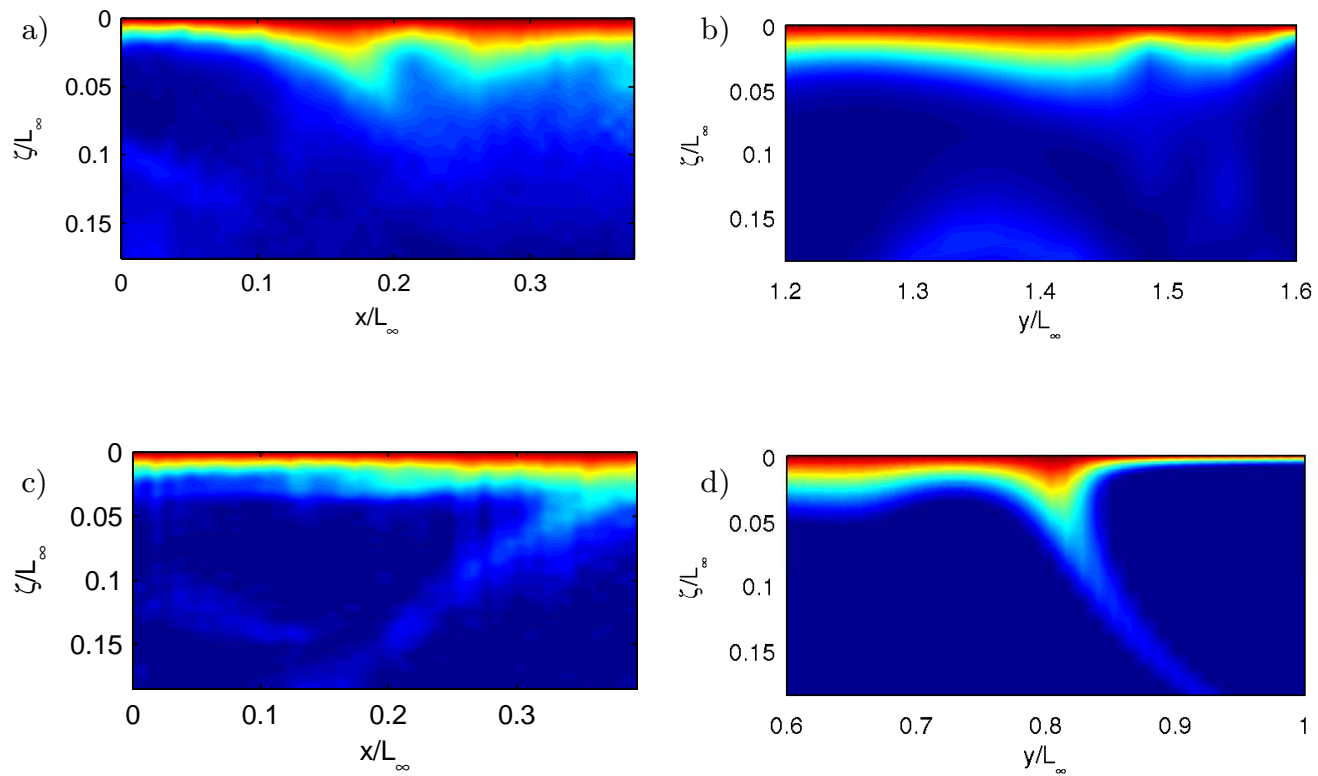

FIGURE 14. Qualitative comparison between laboratory experiments (Herlina \& Jirka 2008) and present DNS results: a) Experiments: $R_{T}=260, S c=500$, b) DNS: $R_{T}=195, S c=500$ (GS200R5), c) Experiments: $R_{T}=520, S c=500$ d) DNS: $R_{T}=507, S c=32$ (GS500). Colour scaling is the same as in Figure 13. 
Figure 16 shows isosurfaces of the concentration at various Schmidt numbers illustrating how the instantaneous concentration boundary layer is affected by the turbulence diffusing from below. In these figures, the isosurfaces represent a concentration of $c=1 / e$ and the colour coding corresponds to the vertical location of the isosurface. The light to dark colour scaling indicates near to far distances to the surface. From $\zeta=0$ to $\delta$, above the shown isosurface, molecular diffusion plays an important role in the scalar transport (see Figure 12b). Moving further down, turbulent transport rapidly takes over the mixing of gas into the bulk. The isosurfaces in Figure 16 and the profiles in Figure 12 illustrate the interchange between molecular diffusion and turbulent transport at various $S c$ numbers.

It can be recognized that the topography of the isosurface at $S c=2$ is more irregular than that of the other isosurfaces at larger Schmidt numbers. This can be explained by the fact that the thickness of the boundary layer $\delta_{e}$ increases with decreasing $S c$. As the vertical location of the isosurface at $S c=2$ is nearest to the turbulence generating source (at the bottom of the computational domain), the turbulence impinging on the isosurface tends to be stronger. With increasing $S c$ the topography becomes smoother as closer to the surface the vertical turbulent velocity rapidly decreases to zero.

The figure also shows that the crests -due to strong downwelling motions- surrounding the divergence area become narrower with increasing $S c$. As explained above, the diverging area itself comprises the strong upwelling area and the area where the flow moves radially outward with low vertical velocity. At $S c=2$ the crests are about $0.5 L_{\infty}$ wide, while at $S c=500$ they are much smaller. Because of the low $R_{T}$ and the fact that the $S c=500$ isosurface is located so close to the interface, only the footprints of the large turbulent eddies can be seen, while any evidence of smaller eddies has virtually disappeared.

Next we examine the effect of different turbulent Reynolds numbers on the topology of the isosurfaces. A sequence of isosurfaces from GS80 and GS500 is presented in Figures 17 and 18, respectively. The $x$ and $y$ coordinates are normalized by $L_{\infty}$ to facilitate the comparison between the two $R_{T}$ cases. The contour maps indicate that also for $R_{T}$ $=507$ the top surface is dominated by divergence areas separated by narrow downwelling (flow-converging) areas. Compared to the $R_{T}=84$ case, an increased number of smaller strucures are present in the $R_{T}=507$ case as is also evidenced by the cumulative spectra discussed below. Compared to what was observed at $R_{T}=84$ (Figure 16), at larger $R_{T}$ not only the largest eddies but also the smaller eddies manage to clearly affect the concentration boundary layer.

Figure 19a presents a snapshot showing velocity vectors together with contours of the $z$-component of the vorticity in a horizontal plane immediately below the interface from simulation GS500. The intense vorticity regions $(|\omega|>2)$ are indicated by the brightest and darkest areas. Comparing Figure 19a and b, we can see that the intense vorticity regions correlate to the region where the concentration boundary layer varies significantly. The significant local thickening of the concentration boundary layer in these regions corresponds to a strong, very localized mass transfer from the surface into the bulk of the fluid.

We can estimate the relative contribution of the different eddy sizes to the total mass flux by plotting the cumulative spectra of the turbulent mass flux $c^{\prime} w^{\prime}$ as shown for GS80 and GS500 in Figures 20a and b, respectively. The spectra of $c^{\prime} w^{\prime}$ are extracted at the concentration boundary layer edge $\left(\right.$ at $\left.\zeta=\delta_{e}\right)$. For all Schmidt numbers the proportion of the turbulent mass flux due to the smaller eddies grows with increasing $R_{T}$. For $S c=2$, for example, the turbulent mass flux due to eddies smaller than the integral length scale is about $7 \%$ at $R_{T}=84$, while at $R_{T}=507$ this proportion is increased to approximately 
a)

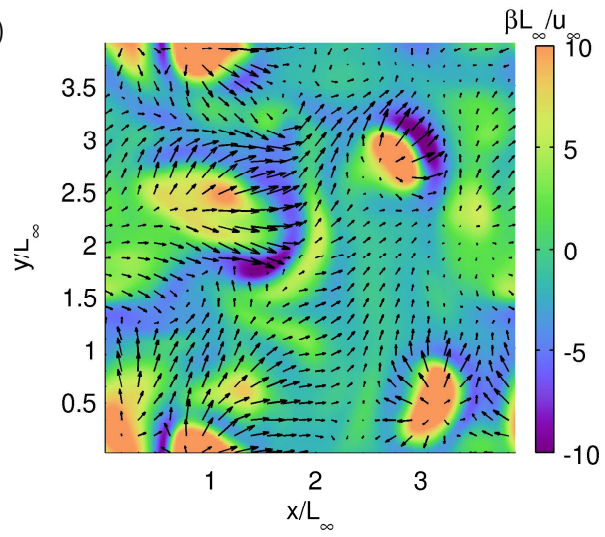

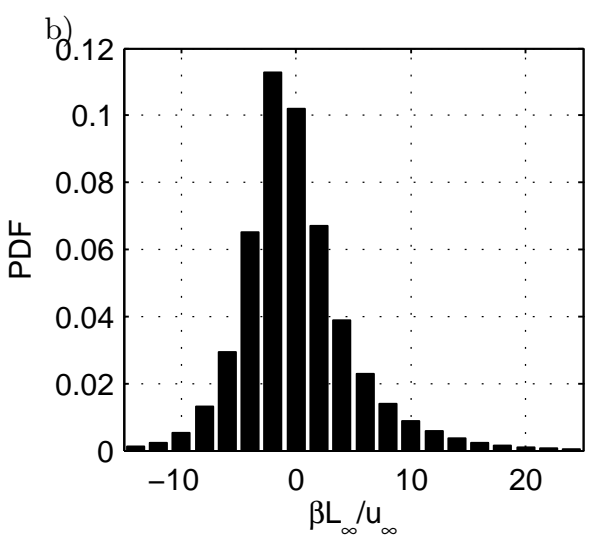

FIgURE 15. Near surface flow structures, Run GS200. a) Horizontal cross-section immediately below the interface showing the surface divergence $(\beta=-\partial w / \partial z)$ contour plot together with the $u-v$ vector-field. b) PDF of surface divergence from all events in GS200.
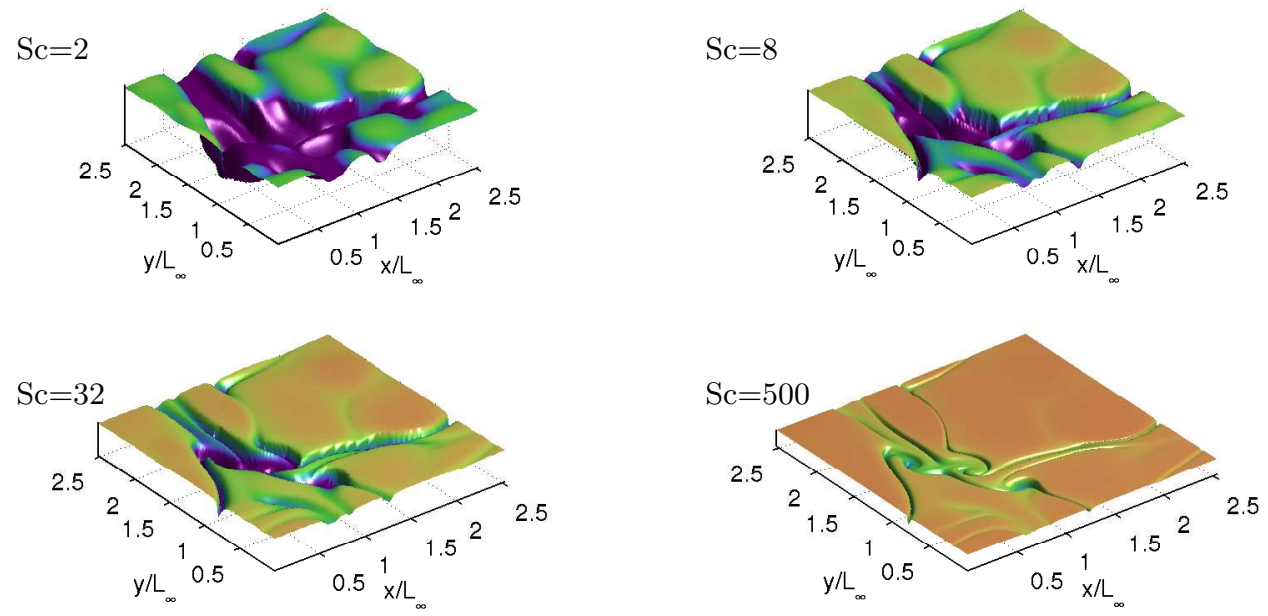

$\zeta / L_{\infty}$

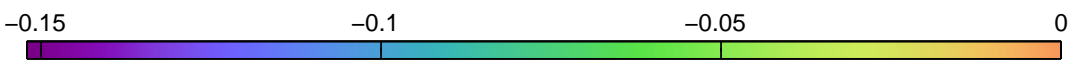

FIGURE 16. 3D isosurface showing position of boundary layer $\delta_{e}$ (where $c=1 / e$ ) at different $S c$ numbers. Each isosurface corresponds to a snapshot taken after $t=30.1 \mathrm{~L} / U$ simulation time (cases GS80 and GS80R5). Twice the thickness of the viscous layer $\left(2 L_{\nu}\right)$ is used as threshold for the darkest color scaling. 

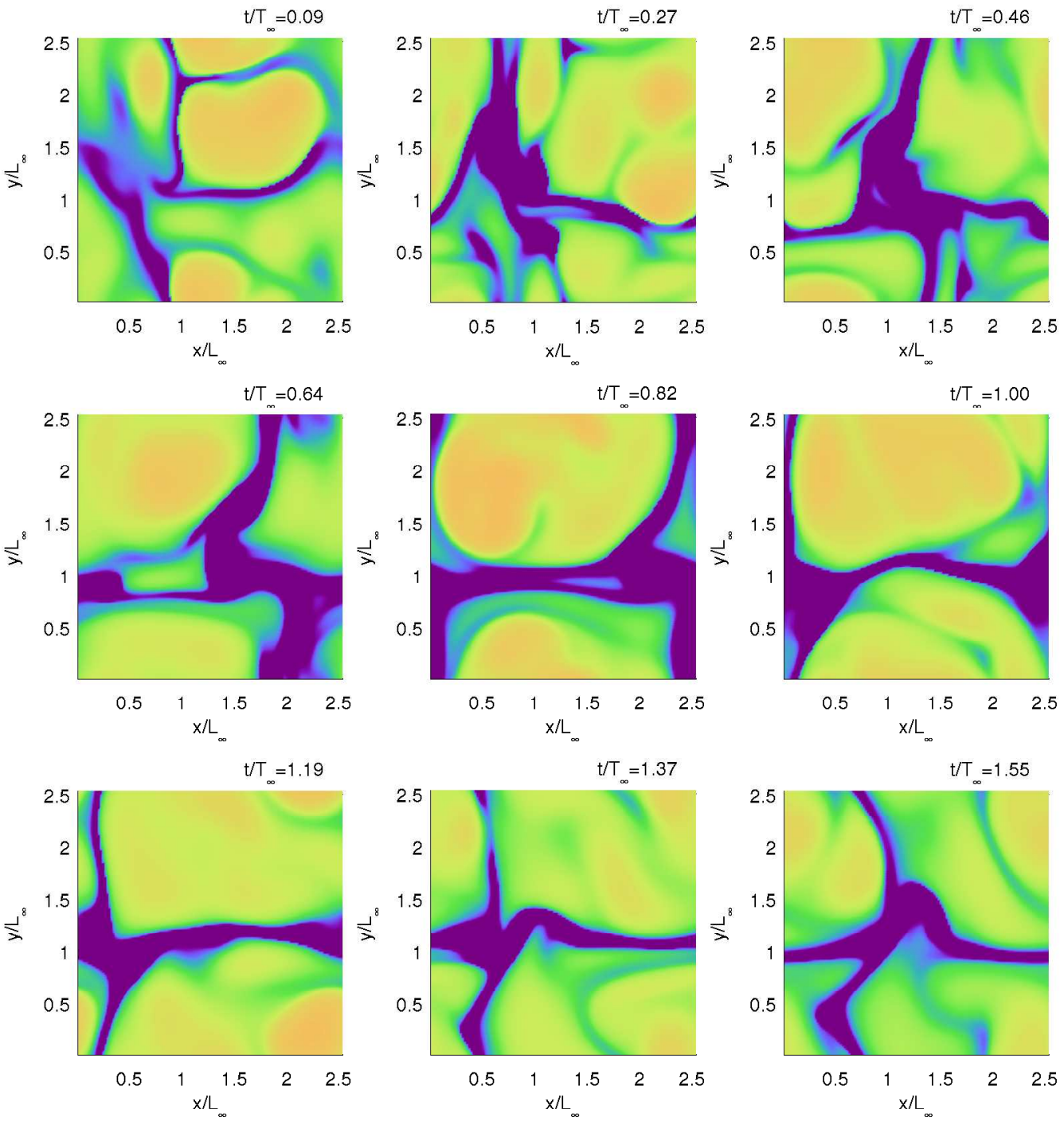

$\zeta / L_{\infty}$

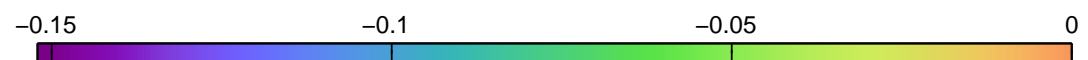

FiguRE 17. Contour map showing position of boundary layer $\delta_{e}$ (where $c=1 / e$ ) at $R_{T}=84$ and $S c=8$ in GS80.

$27 \%$. For the larger Schmidt number of $S c=32$, these proportions change to about $12 \%$ and $30 \%$, respectively. So that for larger $R_{T}$ (and $S c$ ) the contribution to the turbulent mass flux of scales smaller than $L_{\infty}$ can not be neglected.

In Section 5.1 we have shown that in our simulations, where $84<R_{T}<507$, the large 

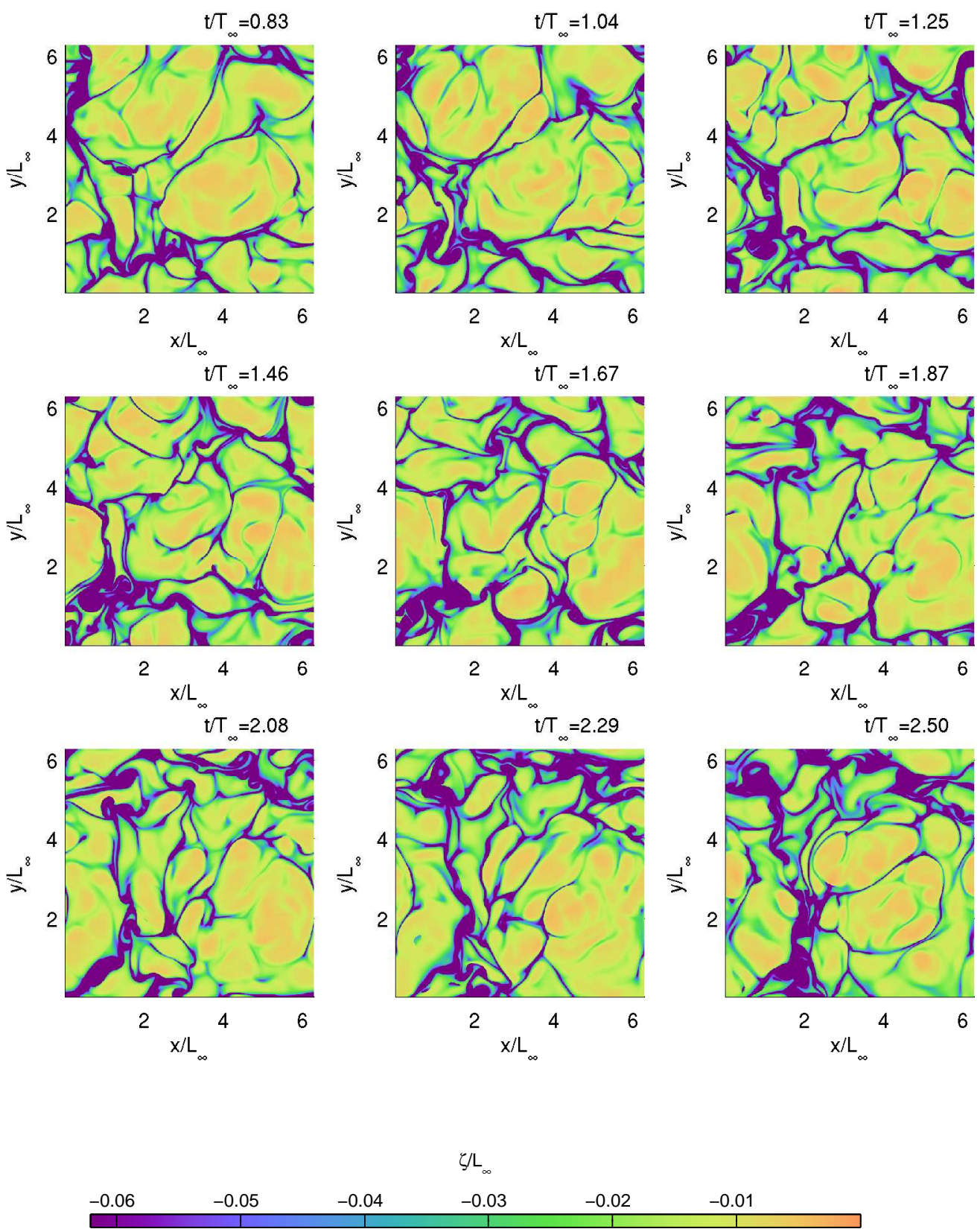

Figure 18. Contour map showing position of boundary layer $\delta_{e}$ (where $c=1 / e$ ) at $R_{T}=507$ and $S c=8$ in GS500.

eddy model of Fortescue \& Pearson (1967) provided a very good estimate of the transfer velocity $K_{L}$ with a coefficient of proportionality of 1.6. However, the results shown in this section indicate that for large $R_{T}$ the smaller eddies start to play an important role in the interfacial mass transfer so that it is expected that for even larger $R_{T}$ the small 

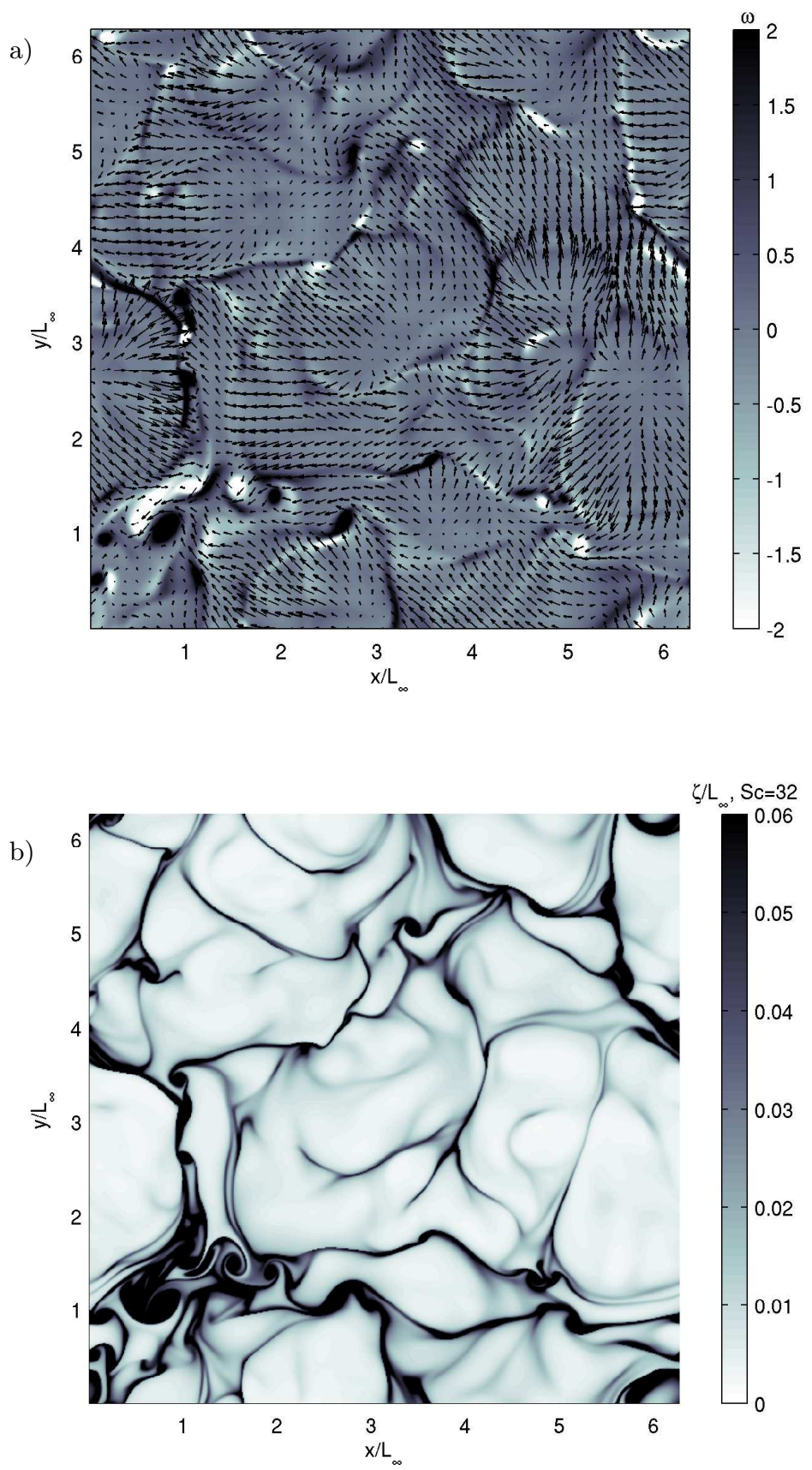

FIGURE 19. Correlation between intense vorticity region and position of concentration boundary layer in GS500. a) Snapshot of the vector-field immediately below the surface with its corresponding $z$-component vorticity contour map and b) Contour map of the concentration boundary layer position (i.e. $\zeta / L_{\infty}$ where $c=1 / e$ ) 

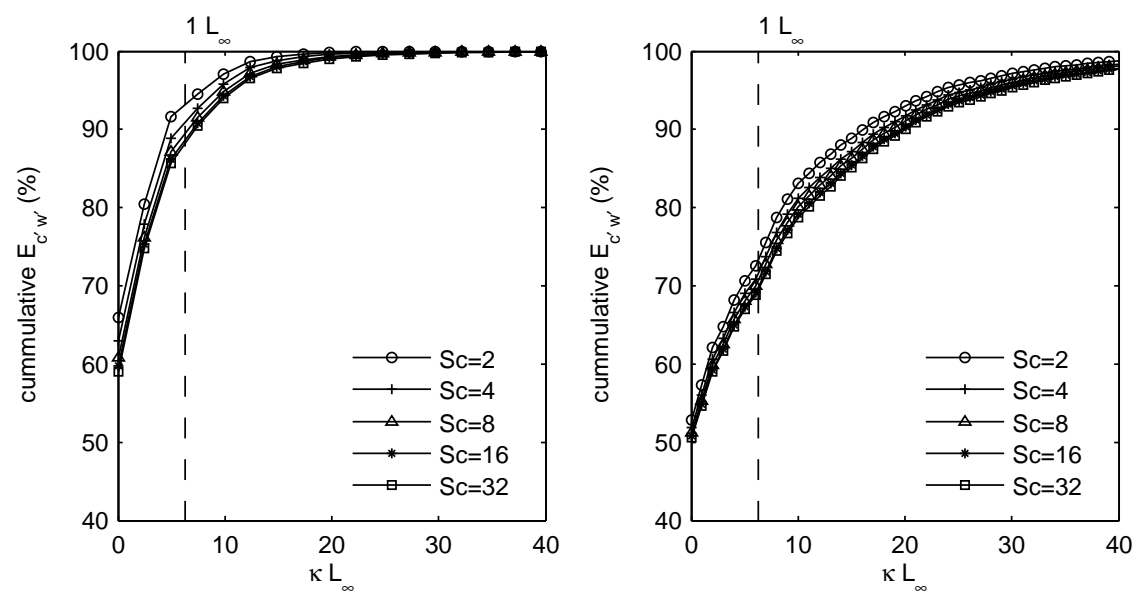

Figure 20. Cumulative energy spectra of $c^{\prime} w^{\prime}$ at $\zeta=5 \delta_{e}$ a) $R_{T}=84$ and b) $R_{T}=507$.

eddy model might give a better estimate of $K_{L}$, which would support the arguments of Theofanous et al. (1976) for the existence of large-eddy and small-eddy regimes for low and high $R_{T}$, respectively.

\section{Conclusions}

Large scale direct numerical simulations (DNS-s) of clean-surface interfacial mass transfer for three different turbulent Reynolds numbers have been performed. A wide range of Schmidt numbers, $2 \leqslant S c \leqslant 500$, were simulated for $R_{T}=84$ and 195. At $R_{T}=507$, however, all $S c$ except $S c=500$ were simulated, the $S c=500$ case was not simulated due to the excessively high demand for computational resources. The interfacial mass transfer was driven by isotropic turbulence diffusing from below. In accordance with grid-stirred experiments, isotropic turbulence was introduced at the bottom of the computational domain to mimic the near-surface effects of the turbulence in open-channel flows. Compared to previously performed simulations of interfacial mass transfer driven by bottom-shear induced turbulence, the present DNSs are the first that provide fully resolved data for realistic Schmidt numbers that are typical for environmental gases like oxygen $(S c=500)$.

Near surface transport processes at small and large Schmidt numbers were found to be qualitatively very similar. The eddies impinging from below were seen to locally either suppress or thicken the concentration boundary layer at the surface. The reduced diffusion at high $S c$ resulted in finer, more localized structures of high scalar concentration.

It was found that the transfer velocity $K_{L}$ scales with $S c^{-1 / 2}$ for the entire range of Schmidt numbers considered. For low to moderate $R_{T}$ it was also found that $K_{L} / u_{\infty} \propto$ $R_{T}^{-1 / 2}$. The above is in agreement with the large eddy model of Fortescue \& Pearson (1967),

A direct comparison of $K_{L}$ obtained in the DNS-s and several grid-stirred experiments with the surface divergence model of McCready et al. (1986) showed a very good agreement when using a constant of proportionality of 0.525 .

For our range of $R_{T}$, the concentration boundary layer thickness was found to be proportional to the outer diffusive sublayer thickness $L_{D}$ and, hence, was determined by 
the size of the largest eddies. For this scaling, the choice of the definition of the boundary layer thickness (either using $\delta_{e}, \delta_{i}$ or $\delta_{c f}$ ) was found to be unimportant, and the variation in the actual thicknesses was found to be small.

The Batchelor thickness, $\delta_{B}$, was found to be proportional to the inner diffusive sublayer (or Batchelor sublayer) thickness $L_{B}$. Note that $L_{B}$ scales with $R_{T}^{-3 / 4}$ rather than with $R_{T}^{-1 / 2}$, which is the typical scaling for the outer diffusive sublayer thickness.

When normalizing the $\zeta$-coordinate (corresponding to the distance from the surface) of the concentration profiles using $\delta_{e}$, an almost perfect collapse of the normalized profiles is observed up to distances of $2 \delta_{e}$ from the surface. Further down, small variations can still be observed which might indicate that apart from the $\delta_{e}$, which is associated with the integral length scales, also smaller scales become important. Similar results were found for the normalization of the $c_{\text {rms }}$ profiles.

While in the experiments it was found to be very difficult to resolve the turbulent mass flux at larger distances from the surface, the present numerical simulations were found to be able to fully resolved the turbulent mass fluxes even in the deeper bulk region. Again, a nice collapse of the profiles is obtained when normalizing the $\zeta$-coordinate using $\delta_{e}$.

Similar to jets impinging on a horizontal plane from below, immediately underneath the surface large divergence areas were found consisting of strong localized upwellings near the centre surrounded by flow moving radially outwards. These divergence areas were found to be separated by small areas with downwelling flow.

Detailed observation of the concentration boundary layer thickness shows that for low $R_{T}$ only the larger eddies are able to distort the boundary layer, while for higher $R_{T}$ also smaller eddies were found to penetrate the boundary layer. The cumulative spectra of the turbulent mass flux indicate that with increasing $R_{T}$ and $S c$ the contribution of the smaller eddies to the total mass flux becomes more significant. Though our data fitted well with the large eddy model, the results obtained at the higher $R_{T}$ indicate that the contribution of the small eddies to the overall mass transfer becomes more significant with increasing $R_{T}$. Hence, it is likely that for even higher $R_{T}$ the small eddy model is more relevant than the large eddy model, supporting the two-regime mass transfer model of Theofanous et al. (1976).

\section{Acknowledgements}

The authors would like to thank the German Research Foundation (DFG) for funding this project and the steering committee of the Super Computing Facilities in Bavaria (HLRB) for granting computing time on the SuperMIG cluster at the Leibniz Computing Centre (LRZ) in Munich. The additional funding by the Helmholtz Water Network is greatly appreciated.

\section{REFERENCES}

Banerjee, S., Lakehal, D. \& Fulgosi, M. 2004 Surface divergence model for scalar exchange between turbulent streams. Intl J. Multiphase Flow 30, 963-977.

Banerjee, S., Rhodes, E. \& Scott, D. S. 1968 Mass transfer to falling wavy liquid films in turbulent flow. Ind. Engng Chem. Fund. 7, 22.

Brumley, B. H. \& JiRKA, G. H. 1987 Near-surface turbulence in agrid-stirred tank. J. Fluid Mech. 183, 235-263.

Brumley, B. H. \& JiRKA, G. H. 1988 Air-water transfer of slightly soluble gases: turbulence interfacial processes and conceptual models. Physo Chem. Hydrodyn. 10 (3), 295-319.

Calmet, I. \& Magnaudet, J. 2003 Statistical structure of high-reynolds-number turbulence close to the free surface of an open-channel flow. J. Fluid Mech. 474, 355-378. 
Chu, C. R. \& JiRKA, G. H. 1992 Turbulent gas flux measurements below the air-water interface of a grid-stirred tank. Intl J. Heat Mass Transfer 35 (8), 1957-1968.

Danckwerts, P. V. 1951 Significance of liquid-film coefficients in gas absorption. Ind. Engng Chem. 43, 1460-1467.

Fortescue, G. E. \& Pearson, J. R. 1967 On gas absorption into a turbulent liquid. Chem. Engng Science 22, 187-216.

GrötzBACH, G. 1983 Spatial resolution requirements for direct numerical simulation of the rayleigh-bènard convection. J. Comp. Phys. 49, 241-264.

Handler, R. A., Saylor, J. R., Leighton, R. I. \& Rovelstad, A. L. 1999 Transport of a passive scalar at a shear-free boundary in fully turbulent open channel flow. Phys. Fluids 11, 2607-2625.

Hasegawa, Y. \& Kasagi, N. 2009 Hybrid dns/les of high schmidt number mass transfer across turbulent airwater interface. Int. J. Heat and Mass Transfer 52, 1012-1022.

HeRlina \& JiRKA, G. H. 2004 Application of LIF to investigate gas transfer near the air-water interface in a grid-stirred tank. Exps in Fluids 37 (3), 341-349.

Herlina \& JiRKa, G. H. 2008 Experiments on gas transfer at the air-water interface induced by oscillating grid turbulence. J. Fluid Mech. 59, 183-208.

HigBie, R. 1935 The rate of absorption of a pure gas into a still liquid during short periods of exposure. AIChE Transactions 31, 365-390.

Hopfinger, E.J. \& Toly, J.A. 1976 Spatially decaying turbulence and its relation to mixing across density interfaces. J. Fluid Mech. 78, 155-175.

Hunt, J. C. R. 1984 Turbulence structure and turbulent diffusion near gas-liquid interfaces. In Gas Transfer at Water Surfaces (ed. W.Brutsaert \& G.H. Jirka), pp. 67-82. Reidel.

Hunt, J. C. R. \& Graham, J. M. R. 1978 Free-stream turbulence near plane boundaries. J. Fluid Mech. 84, 209-235.

Jähne, B. \& Haussecker, H. 1998 Air-water gas exchange. Annu. Rev. Fluid Mech. 30, 443468.

Kermani, A. \& Shen, L. 2009 Surface age of surface renewal in turbulent interfacial transport. Geophysical Research Letters 36, L10605.

Khakpour, H.R., Shen, L. \& Yue, D.K.P. 2011 Transport of passive scalar in turbulent shear flow under a clean or surfactant-contaminated free surface. J. Fluid Mech. 670, 527-557.

Komori, S., Ueda, H., Ogino, F. \& Mizushina, T. 1982 Turbulence structure and transport mechanism at the free surface in an open channel flow. International Journal of Heat and Mass Transfer 25 (4), 513-521.

Kubrak, B., Herlina, H., Greve, F. \& Wissink, J.G. 2013 Low-diffusivity scalar transport using a weno scheme and dual meshing. J. Comput. Phys. 240, 158-173.

Lamont, J. C. \& ScotT, D. S. 1970 An eddy cell model of mass transfer into the surface of a turbulent liquid. AIChE Journal 16, 513-519.

Lewis, W. K. \& Whitman, W.G. 1924 Principles of gas absorption. Ind. Engng Chem. 16.

Liu, X., Osher, S. \& Chan, T. 1994 Weighted essentially non-oscillatory schemes. J. Comp. Physics 115, 200-212.

Lu, D.M. \& Hetsroni, G. 1995 Direct numerical simulation of a turbulent open channel flow with passive heat transfer. Intl J. Heat Mass Transfer 38.

Magnaudet, J. \& CAlmet, I. 2006 Turbulent mass transfer through a flat shear-free surface. J. Fluid Mech. 553, 155-185.

McCready, M.J., Vassiliadou, E. \& Hanratty, T.J. 1986 Computer-simulation of turbulent mass-transfer at a mobile interface. AIChE J. 32 (7), 1108-1115.

McKennA, S. P. \& MCGillis, W.R. 2002 Surface divergence and air-water gas transfer. In Gas Transfer at Water Surfaces (ed. E. S. Saltzman M. A. Donelan, W. M. Drennan \& R. Wanninkhof), Geophysical Monograph, vol. 127, pp. 129-134. American Geophysical Union.

McKenna, S. P. \& McGillis, W.R. 2004a Observations of flow repeatability and secondary circulation in oscillating grid-stirred tank. Phys. Fluids 16 (9), 3499-3502.

McKenna, S. P. \& McGillis, W.R. $2004 b$ The role of free-surface turbulence and surfactants in air-water gas transfer. Int.J.Heat Mass Transfer 47, 539-553.

Moog, D. B. \& JiRkA, G. H. 2002 Air-water gas transfer in uniform flows with large gravelbed roughness. In Gas Transfer at Water Surfaces (ed. E. S. Saltzman M. A. Donelan, W. 
M. Drennan \& R. Wanninkhof), Geophysical Monograph, vol. 127, pp. 371-376. American Geophysical Union.

Münsterer, T., Mayer, H.J. \& JÄHne, B. 1995 Dual-tracer measurements of concentration profiles in the aqueous mass boundary layer. Air-Water Gas Transfer : 3rd International Symposium on Air-Water Gas Transfer pp. 637-648.

NAGAOSA, R. 1999 Direct numerical simulation of vortex structures and turbulent scalar transfer across a free surface in a fully developed turbulence. Phys. Fluids 11, 1581-1595.

O'Connor, D.J. \& DobBins, W.E. 1956 The mechanism of reaeration in natural streams. J. Sanitary Engng 82 (SA6), 1115-1140.

Plate, E. J. \& Friedrich, R. 1984 Reaeration of open channel flow. Gas Transfer at Air-Water Interfaces pp. 333-346.

Pope, S. 2000 Turbulent Flows. Cambridge University Press.

Schladow, S.G., Lee, M., Hürzeler, B.E. \& Kelly, P.B. 2002 Oxygen transfer across the air-water interface by natural convection in lakes. Limnol. Ocean. 47 (5), 1394-1404.

Sugihara, Y. \& Tsumori, H. 2005 Surface-renewal eddies at the air-water interface in oscillating-grid turbulence. Env. Hyd. Sust. Water Management pp. 199-205.

TAmburrino, A. \& Gulliver, J.S. 2002 Free-surface turbulence and mass transfer in a channel flow. AIChE J. 48 (12), 2732-2743.

Theofanous, T. G. 1984 Conceptual models of gas exchange. Gas transfer at air-water surfaces. Reidel. pp. 271-281.

Theofanous, T. G., Houze, R. N. \& Brumfield, L. K. 1976 Turbulent mass transfer at free, gas liquid interfaces with applications to open channel, bubble and jet flows. Intl J. Heat Mass Transfer 19.

Turney, D. E. \& BAnERJeE, S. 2013 Air-water gas transfer and near-surface motions. J. Fluid Mech. 733, 588-624.

Walker, D. T., Leighton, R. I. \& Garza-Rios, L. O. 1996 Shear-free turbulence near a flat free surface. J. Fluid Mech. 320, 19-51.

WAlker, J.W. \& PeIRson, W.L. 2008 Measurement of gas transfer across wind-forced wavy airwater interfaces using laser-induced fluorescence. Exp. Fluids 44, 249-259.

Wissink, J.G. 2004 On unconditional conservation of kinetic energy by finite-difference discretisations of the linear and non-linear convection equation. Computers and Fluids $\mathbf{3 3}$, $315-343$.

WolfF, L. M. \& HANRATTy, T. J. 1994 Instantaneous concentration profiles of oxygen accompanying absorption in a stratified flow. Exps Fluids 16, 385-392.

Woodrow, P. T. \& Duke, S. R. 2002 LIF measurements of oxygen concentration gradients along flat and wavy air-water interfaces. In Gas Transfer at Water Surfaces (ed. E. S. Saltzman M. A. Donelan, W. M. Drennan \& R. Wanninkhof), Geophysical Monograph, vol. 127, pp. 83-88. American Geophysical Union.

Yamamoto, Y., Kunugi, T \& Serizawa, A. 2001 Turbulence statistics and scalar transport in an open-channel flow. J. Turbulence $\mathbf{2}$. 